\title{
Structural basis for HCMV Pentamer recognition by antibodies and neuropilin 2
}

Daniel Wrapp ${ }^{{ }^{\star}}$, Xiaohua $\mathrm{Ye}^{2^{\star}}$, Zhiqiang $\mathrm{Ku}^{2}$, Hang Su${ }^{2}$, Harrison G. Jones ${ }^{1}$, Nianshuang Wang ${ }^{1}$, Akaash

K. Mishra ${ }^{1}$, Daniel C. Freed ${ }^{3}$, Fengsheng $\mathrm{Li}^{3}$, Aimin Tang ${ }^{3}$, Leike $\mathrm{Li}^{2}$, Dabbu Kumar Jaijyan ${ }^{4}$, Hua Zhu ${ }^{4}$, Dai Wang ${ }^{3}$, Tong-Ming Fu², Ningyan Zhang², Zhiqiang An", Jason S. McLellan ${ }^{1 \#}$

${ }^{1}$ Department of Molecular Biosciences, The University of Texas at Austin, Austin, TX 78712, USA.

${ }^{2}$ Texas Therapeutics Institute, Brown Foundation Institute of Molecular Medicine, University of Texas

Health Service Center at Houston, Houston, TX 77030, USA.

${ }^{3}$ Merck Research Laboratories, Merck \& Co., Inc., Kenilworth, NJ 07033, USA.

${ }^{4}$ Department of Microbiology, Biochemistry and Molecular Genetics, Rutgers New Jersey Medical School, Newark, NJ 07103, USA.

*These authors contributed equally

${ }^{\#}$ Correspondence to zhiqiang.an@uth.tmc.edu (Z.A.) and jmclellan@austin.utexas.edu (J.S.M.) 


\begin{abstract}
Human cytomegalovirus (HCMV) encodes for multiple surface glycoproteins and glycoprotein complexes ${ }^{1,2}$. One of these complexes, the HCMV Pentamer ( $\mathrm{gH}, \mathrm{gL}, \mathrm{UL} 128, \mathrm{UL} 130$ and UL131), mediates tropism to both epithelial and endothelial cells by interacting with the cell surface receptor neuropilin 2 (NRP2) $)^{3,4}$. Despite the critical nature of this interaction, the molecular determinants that govern NRP2 recognition remain unclear. Here we describe the cryo-EM structure of NRP2 bound to the HCMV Pentamer. The high-affinity interaction between these proteins is calcium-dependent and differs from the canonical C-terminal arginine (CendR) binding that NRP2 typically utilizes ${ }^{5,6}$. The interaction is primarily mediated by NRP2 domains a2 and b2, which interact with UL128 and UL131. We also determine the structures of four human-derived neutralizing antibodies in complex with the HCMV Pentamer to define susceptible epitopes. The two most potent antibodies recognize a novel epitope yet do not compete with NRP2 binding. Collectively, these findings provide a structural basis for HCMV tropism and antibody-mediated neutralization, and serve as a guide for the development of HCMV treatments and vaccines.
\end{abstract}


Human cytomegalovirus (HCMV) is a ubiquitous pathogen and congenital infection can cause debilitating and permanent birth defects ${ }^{7-9}$. Despite the severity of these infections and the prevalence of this pathogen, there are currently no FDA-approved vaccines and therapeutic options are limited ${ }^{10-12}$. HCMV is an enveloped, double-stranded DNA virus of the family Herpesviridae ${ }^{13}$. The surface of the viral membrane is decorated by several glycoprotein complexes that mediate viral entry and membrane fusion ${ }^{14-16}$. One of these complexes is the HCMV Trimer, composed of glycoproteins $\mathrm{gH}, \mathrm{gL}$, and $\mathrm{gO}^{2,17}$. The HCMV Trimer mediates tropism for fibroblasts by binding platelet derived growth factor receptor alpha (PDGFR $\alpha)^{18,19}$. The HCMV Trimer is also capable of mediating infection of a broader variety of cell types by interacting with transforming growth factor beta receptor 3 (TGF $\beta R 3)^{4,20}$. The other critical tropism-determining complex is the HCMV Pentamer, which is composed of glycoproteins UL128, UL130, UL131, and the same $\mathrm{gH}$ and $\mathrm{gL}$ proteins that comprise the bulk of the HCMV Trimer ${ }^{1,3}$. This elongated heteropentamer mediates tropism for endothelial and epithelial cells by binding to neuropilin 2 (NRP2) and triggering the viral fusion protein, $\mathrm{gB}$, to facilitate viral entry into host cells $\mathrm{s}^{4,15,21-23}$.

Neuropilins 1 and 2 are single-pass transmembrane proteins that are expressed on the surface of neuronal, epithelial, and endothelial cells ${ }^{24,25}$. Under normal conditions, these proteins function as receptors and co-receptors that engage in numerous physiological processes, including angiogenesis and development of the nervous system ${ }^{26,27}$. NRP2 is composed of two N-terminal CUB domains (a1 and a2), two F5/8 domains (b1 and b2), a MAM domain, a transmembrane domain, and a C-terminal PDZ domain that is thought to mediate cytoplasmic signaling in response to extracellular stimuli ${ }^{28,29}$. Perhaps the most thoroughly characterized of these stimuli is vascular endothelial growth factor (VEGF) ${ }^{30}$. The crystal structure of these proteins in complex with one another has been determined, revealing that the b1 domain of NRP2 engages the C-terminal arginine of VEGF ${ }^{6,31}$. Since this initial characterization, the NRP2 b1 domain has been shown to interact with other binding partners via the same mechanism², prompting the moniker "CendR" to refer to this exposed C-terminal arginine motif5. Although it has been shown that soluble NRP2 is capable of inhibiting HCMV infection of epithelial cells ${ }^{4}$, the molecular determinants that mediate this interaction remain unclear, and several additional Pentamer receptors have been proposed ${ }^{33,34}$. The most potently neutralizing HCMV-directed antibodies are elicited against 
the Pentamer, suggesting that it represents a susceptible target for the development of vaccines and immunotherapeutics ${ }^{35-37}$.

To investigate NRP2 and mAb binding, we initiated structural and biophysical studies. Based on

32 previous crystallographic experiments that reported conserved calcium-coordinating loops in both the a1

33 and a2 domains of NRP2 ${ }^{38,39}$, we measured the affinity of recombinantly expressed NRP2 a1a2b1b2 for

34 the soluble HCMV Pentamer ectodomain in both the presence and absence of calcium. We found that in

35 the presence of 2 mM EDTA, no association between NRP2 and Pentamer could be detected. However, when the same experiment was performed in the presence of $2 \mathrm{mM} \mathrm{CaCl}_{2}$, the affinity of the interaction

37 was determined to be $2.2 \mathrm{nM}$ (Supplementary Fig. 1a-b). It is possible that a failure to add additional

38 calcium is what necessitated the use of chemical cross-linkers during previous efforts to observe this complex by negative-stain electron microscopy ${ }^{4}$. The addition of $2 \mathrm{mM} \mathrm{CaCl}_{2}$ enabled us to form a stable $\sim 230 \mathrm{kDa}$ complex that was suitable for cryo-EM screening. The addition of $0.1 \%$ amphipol A8-35 helped to prevent aggregation and allowed for the determination of a $4.0 \AA$ resolution cryo-EM structure of the

42 HCMV Pentamer bound by human NRP2 (Fig. 1a, Supplementary Figs. 2 and 3). Performing focused

43 refinement on the NRP2-bound UL proteins yielded a $3.65 \AA$ reconstruction that aided in model building

44 and refinement.

These reconstructions revealed an extensive binding interface, with contacts formed by NRP2

47 forms a sizable portion of this binding interface, likely providing an explanation as to why high-affinity

48 NRP2 binding could only be observed after the addition of $2 \mathrm{mM} \mathrm{CaCl}_{2}$ (Fig. 1a-b). Additional contacts

49 are formed between the C-terminal beta strands of ULs 130 and 131 and a loop formed by residues 453-

50461 of the b2 domain of NRP2 (Fig. 1c). This mode of NRP2 binding differs from the canonical CendR

51 motif binding that has been described previously for other NRP2-binding partners ${ }^{40,41}$. The CendR binding

52 mechanism involves the engagement of a C-terminal arginine residue by the b1 domain, whereas

53 Pentamer is exclusively bound by the a1, a2, and b2 domains. Furthermore, none of the three UL

54 proteins contain a positively charged C-terminal arginine that makes up the CendR motif. As expected,

55 the majority of the binding interface from the Pentamer is composed of the tropism-determining UL

56 proteins, particularly UL128 and UL $131^{1,3}$, which respectively contribute $437.5 \AA^{2}$ and $208.4 \AA^{2}$ of buried 
57 surface area to the interface. Whereas the NRP2 a2, b1, and b2 domains are clustered tightly together at 58 the head of the Pentamer, the N-terminal a1 domain is tethered via a flexible linker that allows it to bind

59 near the middle of the Pentamer, where the C-terminus of UL128 associates with gL. The local resolution 60 for this portion of the reconstruction was relatively poor compared to the rest of the complex, suggesting 61 either conformational flexibility in this region or a loose association of a1. To test the importance of the a1 62 domain, we expressed NRP2 with a 144-residue N-terminal truncation and observed that even in the 63 absence of this flexibly tethered a1 domain, NRP2 a2b1b2 was capable of binding to the HCMV

64 Pentamer with $7.9 \mathrm{nM}$ affinity (Supplementary Fig. 1c), supporting our structural observations that the 65 critical determinants of Pentamer binding are contained within NRP2 domains a2b1b2. Intriguingly, our 66 cryo-EM data processing also revealed that a second, more poorly resolved copy of NRP2 could be 67 observed binding near the C-terminal arginine of $\mathrm{gL}$ via the b1 domain (Supplementary Figs. 2 and 4).

68 Although this second NRP2 appears to exhibit the canonical CendR binding, it could only be observed in $69 \sim 40 \%$ of particles. Furthermore, its binding to the gL protein rather than the tropism-determining UL 70 proteins suggests that this second copy of NRP2 is likely an artifact of the high concentrations of NRP2

71 that were used to form a stable complex. Overall, the conformation of the receptor-bound Pentamer 72 ectodomain does not drastically differ from that of the unbound Pentamer ${ }^{42}$ (Supplementary Fig. 5),

73 suggesting that rather than undergoing substantial conformational rearrangements, this complex acts as

74 a tether to connect HCMV virions to the surface of epithelial and endothelial cells until the viral fusogen

75 gB fuses the viral and cellular membranes.

Previous efforts to characterize the humoral immune response to asymptomatic HCMV infection

77 yielded an extensive panel of neutralizing antibodies directed against $\mathrm{gB}$, the HCMV Trimer, and the

78 HCMV Pentamer ${ }^{35,43,44}$. To learn more about the mechanisms of neutralization of high-affinity, Pentamer-

79 directed antibodies, we determined cryo-EM structures of four naturally elicited human antibodies in

80 complex with the Pentamer (Fig. 2, Supplementary Figs. 3, 6, 7, and 8). The flexibility and elongated

81 shape of the Pentamer necessitated focused refinements of the Fabs along with the domains making up

82 their respective epitopes. Model building was facilitated by high-resolution crystal structures of unbound

83 Fabs (1-103: $1.9 \AA, 1-32: 2.1 \AA, 2-18: 2.8 \AA, 2-25: 2.5 \AA)$, which were then used as reference restraints

84 and lightly refined as a part of the complex (Supplementary Tables 1 and 2). Three of these antibodies 
$85(1-103,2-18$, and 2-25) bound solely to the UL proteins at the head of the Pentamer, whereas the fourth

86 (1-32) bound to $\mathrm{gL}$, near the junction between the UL proteins and the conserved $\mathrm{gH} / \mathrm{gL}$ scaffold (Fig.

87 2a). The Fab 1-103 epitope is solely composed of residues from the membrane-distal tip of UL128,

88 sometimes referred to as Site 1 of immunogenic region (IR) ${ }^{4,35}$. The epitopes of Fabs $2-18$ and 2-25

89 overlap substantially, with both Fabs binding to the junction between ULs 128 and 131. This junction

90 where UL128 meets UL131 does not fit into one of the preexisting antigenic sites, but rather overlaps with

91 both Site 2 and Site 5 of IR14,35. The Fab 1-32 epitope spans the interface between gH and gL, slightly

92 below Site $4 / 6$ in IR2 $2^{4,35}$ (Fig. 2b). This epitope is consistent with previous observations ${ }^{35}$ that $1-32$ is the

93 only one of the four antibodies evaluated that was capable of binding to both the fully assembled HCMV

94 Pentamer and disulfide-linked homodimers of the $\mathrm{gH} / \mathrm{gL}$ heterodimer. Despite the ability to recognize the

$95 \mathrm{gH} / \mathrm{gL}$ heterodimer that serves as the scaffold for assembly of both the HCMV Pentamer and Trimer, 1-32

96 was only capable of neutralizing HCMV infection in epithelial cells ${ }^{35}$.

97 By analyzing the structures of these immunocomplexes in conjunction with the structure of

98 Pentamer bound by NRP2, it becomes possible to delineate more clearly the molecular basis for

99 neutralization (Fig. 3). The CDR H3 and CDR L1 of Fab 1-103 compete with NRP2 by binding to the

100 same portion of Pentamer that is engaged by the calcium-coordinating loop (residues 251 to 258 ) of

101 NRP2 domain a2. By binding to the junction between $\mathrm{gL}$ and the UL head of Pentamer, Fab 1-32

102 occupies the same space as several of the loops of the a1 domain of NRP2 (residues 45-48; 72-77;

103 106-110) via its CDR L1 (Fig. 3b). Consistent with the relatively poor density for domain a1, we found

104 that when bound by 1-32, Pentamer was still capable of interacting with NRP2, albeit with diminished

105 affinity (133 nM) (Supplementary Fig. 9), further supporting our findings that the a1 domain is not strictly

106 required for binding to Pentamer. This observation is also consistent with the relatively weak

107 neutralization capacity of 1-32 compared to 1-103, which competes for the a2b1 b2 interface.

108 Intriguingly, the two most potently neutralizing mAbs that we examined, 2-18 and 2-25 (Fig. 4a),

109 do not appear to compete with NRP2 a1a2b1b2 based on our structural analysis. Furthermore, although it

110 is possible that 2-18 and 2-25 compete with the C-terminal MAM domain, this seems unlikely based on

111 the position of the C-terminus of $\mathrm{b} 2^{45}$. These mAbs are both directed against an epitope at the junction

112 between ULs 128 and 131, and although this epitope is directly adjacent to the NRP2 binding interface, 
113 the binding angles of 2-18 and 2-25 result in these two Fabs being directed away from the $\beta$-sheet-rich

114 face of the Pentamer that is responsible for engaging NRP2. A biolayer interferometry-based competition

115 assay confirmed our structural observation that Fabs 2-18 and 2-25 do not disrupt the interaction between

116 Pentamer and NRP2 a1a2b1b2 (Fig. 4b). These two Fabs are still capable of neutralizing HCMV in vitro

117 even when administered up to 30 minutes after allowing viruses to adhere to human epithelial cells,

118 although we observed a significant decrease in the neutralization potency of 2-25 Fab, as compared to 2-

11925 IgG (Figs. 4a, 4c). This broad and potent neutralization of multiple HCMV strains (Fig. 4d), which

120 occurs without disrupting the interaction between NRP2 a1a2b1b2 and the Pentamer, suggests that 2-18

121 and 2-25 neutralize via a distinct mechanism from 1-103 and 1-32 (Supplementary Fig. 10). Whether 2-

12218 and 2-25 prevent the association of an unidentified secondary receptor or prevent some

123 conformational change that is required to trigger gB-induced membrane fusion remains unclear and

124 requires additional investigation.

125 Collectively, these data provide a molecular basis of HCMV tropism for both epithelial and

126 endothelial cells. Due to the importance of these cell types during natural infection, this represents a

127 critical advance in our understanding of how HCMV engages host cells at one of the earliest stages of

128 infection ${ }^{22,46}$. Similarly, the structure of the HCMV Trimer was recently reported in complex with PDGFR $\alpha$

129 and TGF $\beta$ R3, two host cell receptors that both mediate tropism of fibroblasts ${ }^{18,20}$. In an effort to explain

130 how this interaction might lead to triggering of $\mathrm{gB}$ and viral fusion, the authors speculate that receptor

131 engagement of the Trimer may induce a rigid-body rotation relative to the viral membrane that causes the

132 attachment complex to destabilize prefusion $\mathrm{gB}^{47}$. Our findings agree with their observation that receptor

133 binding does not induce any conformation changes, lending credence to the hypothesis that a rigid-body

134 rotation of the receptor-binding complex may act as trigger to induce membrane fusion. However, the

135 existence of neutralizing mAbs that do not disrupt NRP2 binding suggests that additional fusion triggers,

136 perhaps in the form of secondary receptors ${ }^{33,34}$ may exist, necessitating further investigation.

137 In addition to detailing the molecular determinants that mediate HCMV infection, these findings

138 expand our understanding of how antibody-mediated neutralization of HCMV can be achieved. Previous

139 structural work has delineated a series of antigenic sites covering the surface of the Pentamer ${ }^{4,35,42,48}$, but

140 in the absence of high-resolution information regarding the NRP2 binding interface, the mechanisms of 

available under aCC-BY-NC-ND 4.0 International license.

141 neutralization for antibodies targeting these sites were unknown. Our data suggest that it may be possible

142 to neutralize HCMV via multiple, distinct mechanisms simultaneously by administering a cocktail of

143 Pentamer-directed antibodies ${ }^{36,49,50}$. By elucidating which epitopes on the surface of the Pentamer are

144 susceptible to antibody-mediated neutralization, these findings will also help to guide future structure-

145 based vaccine design efforts. 


\section{ACKNOWLEDGEMENTS}

We thank Emilie Shipman and Dr. John Ludes-Meyers for their assistance with cell transfection

149 and protein production; Drs. Aguang Dai and Sasha Dickinson at the Sauer Structural Biology Laboratory

150 for their assistance with microscope alignment and data collection; the 19-ID beamline staff at the Structural

151 Biology Center at the Advanced Photon Source, Argonne National Laboratory; and Dr. Georgina Salazar

152 for assistance with manuscript preparation. This study was funded in part by grants from Merck \& Co., Inc.

153 Kenilworth, NJ, USA, the Texas Emerging Technology Fund, and the Welch Foundation Grant No. AU-

154 0042-20030616. The Sauer Structural Biology Laboratory is supported by the University of Texas College

155 of Natural Sciences and by award RR160023 from the Cancer Prevention and Research Institute of Texas

156 (CPRIT). Argonne National Laboratory is operated by UChicago Argonne, LLC, for the U.S. Department of

157 Energy (DOE), Office of Biological and Environmental Research under Contract DE-AC02-06CH11357.

\section{AUTHOR CONTRIBUTIONS}

D.W., X.Y., Z.A., and J.S.M. conceived of and designed experiments. D.W., X.Y., H.G.J., N.W.,

160 and A.K.M. produced and purified proteins. D.W., H.G.J., and A.K.M. performed crystallographic studies.

161 D.W. performed BLI and SPR experiments. D.W. and J.S.M. collected and analyzed cryo-EM data. X.Y.,

162 Z.K., and L.L. established the neutralization assays and X.Y., H.S., D.C.F., and F.L. performed the

163 assays. X.Y., Z.K., A.T., and D. Wang analyzed the neutralization data. D.K.J. and H.Z. prepared the

164 HCMV AD169rev-GFP virus. Z.A., T-M.F., N.Z., and J.S.M. supervised experiments. D.W., X.Y., Z.A., and

165 J.S.M. wrote the manuscript with input from all authors. 
172 Figure 1: The cryo-EM structure of the HCMV Pentamer bound by NRP2. (a) Cryo-EM density is shown

173 (left), with the Pentamer colored in shades of green, gray and white and NRP2 colored orange. The

174 atomic model of this complex (right) is shown as ribbons, with the NRP2 also represented by a

175 transparent molecular surface. (b) The interface between the NRP2 a2 domain and UL128. UL128 is

176 depicted as a transparent, green molecular surface with ribbons underneath and NRP2 is shown as

177 orange ribbons. Residues that are predicted to form critical contacts are shown as sticks. Oxygen,

178 nitrogen, and sulfur atoms are colored red, blue, and yellow, respectively. The single calcium atom is

179 shown as a bright green sphere, with black dotted lines depicting the interaction with conserved

180 coordinating residues. (c) The interface between the NRP2 b2 domain and the HCMV Pentamer. ULs 130

181 and 131 are shown as a transparent, green molecular surface and NRP2 is shown as orange ribbons,

182 with residues predicted to form critical contacts shown as sticks. Oxygen and nitrogen atoms are colored

183 red and blue, respectively.

185 Figure 2: Composite of cryo-EM structures of Pentamer bound by four neutralizing human antibodies. (a)

186 The atomic models of two cryo-EM structures of antibodies bound to the HCMV Pentamer are

187 superimposed based on the position of the UL proteins. The Pentamer is shown as a molecular surface,

188 colored according to Fig. 1 and Fabs are shown as ribbons surrounded by a transparent molecular

189 surface. Fab 1-103 is colored blue, Fab 1-32 is colored gold, Fab 2-18 is colored purple and Fab 2-25 is

190 colored red. (b) CDRs from each Fab are shown as ribbons and the Pentamer is shown as a transparent,

191 molecular surface with ribbons underneath. Predicted critical contact residues are shown as sticks. Fab 1-

192103 (top left) is colored blue, Fab 1-32 (bottom left) is colored gold, Fab 2-18 (top right) is colored purple

193 and Fab 2-25 (bottom right) is colored red. Oxygen, nitrogen and sulfur atoms are colored red, blue and

194 yellow respectively.

196 Figure 3: Pentamer-directed antibodies can neutralize HCMV via multiple mechanisms. (a) Cryo-EM

197 structures of NRP2-bound Pentamer and Fab-bound Pentamer are superimposed based on the position

198 of the UL proteins. Pentamer is shown as a molecular surface colored according to Fig. 1, Fabs are 
shown as ribbons, colored according to Fig. 2, and NRP2 is shown as orange ribbons surrounded by a transparent molecular surface. (b) Close-up views of each Fab-bound Pentamer are superimposed upon the NRP2-bound Pentamer. Both Fabs and NRP2 are shown as ribbons surrounded by a transparent molecular surface, while the Pentamer is shown as a solid molecular surface. NRP2 is colored orange, 1103 is colored blue, 1-32 is colored gold, 2-18 is colored purple and 2-25 is colored red.

Figure 4: Antibodies 2-18 and 2-25 potently neutralize HCMV without disrupting NRP2 binding. (a) Neutralization curves are shown for each mAb based on inhibition of AD169rev-GFP infection in ARPE19 cells. Inhibitory curves for both IgG and Fab are shown, with IgG shown in darker colors. (b) Sensorgrams from a BLI-based competition experiment are displayed. NRP2 was immobilized to a BLI sensor and dipped into Pentamer alone or Pentamer incubated with a molar excess of indicated Fab. (c)

210 IgG neutralization of HCMV post-attachment to epithelial cells. AD169rev-GFP virions were allowed to 211 adhere to ARPE-19 cells and saturating concentrations of IgG were added after a variety of different

212 incubation periods. Viral inhibition is plotted for each $\lg G$ after either 0 mins incubation, 30 mins

213 incubation, or 60 mins incubation. (d) Neutralization potency of 2-18 lgG was evaluated against twelve 214 clinical isolates and two laboratory-adapted HCMV strains in ARPE-19 cells. IC 50 values were calculated

215 by non-linear fit of the percentage of viral inhibition vs. concentration $(\mathrm{ng} / \mathrm{mL})$. The neutralization results of 216 mAbs 1-103, 1-32 and 2-25 against the same panel of HCMV strains have been reported previously ${ }^{35}$.

218 Supplementary Figure 1: The interaction between NRP2 and Pentamer is calcium-dependent. (a) BLI 219 sensorgram showing the absence of binding between Pentamer and NRP2 in the presence of $2 \mathrm{mM}$ 220 EDTA. (b) BLI sensorgram showing binding between Pentamer and NRP2 in the presence of $2 \mathrm{mM}$ 221 calcium. Data are shown as black lines and best fit of a 1:1 binding model is shown as red lines. (c) BLI 222 sensorgram showing binding between Pentamer and NRP2 a2b1b2 in the presence of 2 mM calcium.

223 Data are shown as black lines and best fit of a 1:1 binding model is shown as red lines.

225 Supplementary Figure 2: Pentamer + NRP2 cryo-EM data processing workflow. 
227 Supplementary Figure 3: Cryo-EM structure validation. (a) FSC curves are shown for focused

228 refinements of Pentamer bound by NRP2 (left), Pentamer bound by 1-103, 1-32 and 2-25 (middle) and

229 Pentamer bound by 2-18 and 8I21 (right). (b) Cryo-EM maps from each focused refinement are shown,

230 colored according to local resolution. (c) Portions of each cryo-EM map are shown, with the

231 corresponding atomic models docked into the density. Residue numbering corresponds to UL130 (left,

232 middle) and UL131 (right). (d) Viewing Direction Distribution charts from cryoSPARC are shown for each

233 focused refinement.

235 Supplementary Figure 4: A subset of particles display a second copy of NRP2 bound to the C-terminus 236 of gL. (a) Two-dimensional class averages of Pentamer bound by two copies of NRP2 are shown. (b) A

$237 \sim 4.2 \AA$ cryo-EM reconstruction of Pentamer bound by two copies of NRP2 is shown as a transparent

238 surface. Atomic models of each component are docked in, shown as ribbons. Both copies of NRP2 are 239 colored orange and Pentamer is colored white, except for gL, which is colored blue-to-red from the N-

240 terminus to the C-terminus. The a1 domain from the gL-bound copy of NRP2 was excluded because it

241 could not clearly be resolved in this reconstruction.

243 Supplementary Figure 5: NRP2 binding does not alter the conformation of Pentamer. (a) Previously

244 reported crystal structures of Pentamer ${ }^{42}$ (PDB IDs: 5VOB and 5VOD) are aligned to the cryo-EM structure

245 of the NRP2-bound Pentamer, based on the position of $\mathrm{gH}$. 5V0B is colored blue, 5V0D is colored red

246 and NRP2-bound Pentamer is colored yellow. (b) A previously reported crystal structure of NRP2 ${ }^{38}$ (PDB

247 ID: 2QQK) is aligned to the cryo-EM structure of Pentamer-bound NRP2, based on the position of the b1

248 domain. 2QQK is colored purple and Pentamer-bound NRP2 is colored orange.

250 Supplementary Figure 6: Pentamer + 1-103 + 1-32 + 2-25 cryo-EM data processing workflow.

252 Supplementary Figure 7: Pentamer + 2-18 + 8I21 cryo-EM data processing workflow. 
254 Supplementary Figure 8: Binding kinetics of Pentamer-directed antibodies. SPR sensorgrams showing

255 binding of each of the four neutralizing Fabs are displayed, with data shown as black lines and the best fit

256 of a 1:1 binding model shown as red lines.

258 Supplementary Figure 9: NRP2 binding to Pentamer is partially disrupted by the presence of 1-32.

259 Sensorgrams are shown for an experiment in which 1-32 IgG was immobilized to a BLI sensor, then used

260 to capture Pentamer before being dipped into NRP2. Data for the association and dissociation of NRP2

261 are shown as black lines and the lines of best fit of a 1:1 binding model are shown as red lines.

263 Supplementary Figure 10: Pentamer-directed mAbs neutralize via distinct mechanisms. A cartoon is

264 shown depicting the infection of an endothelial or epithelial cell by HCMV. Pentamer is colored according

265 to Fig. 1, NRP2 is colored orange and the three protomers of gB are colored red, blue and yellow. The

266 stages of infection that mAbs 1-103, 1-32, 2-18 and 2-25 are predicted to disrupt are denoted by inhibition

267 arrows.

269 Supplementary Table 1: X-ray crystallographic data collection and refinement.

271 Supplementary Table 2: Cryo-EM data collection and refinement. 


\section{METHODS}

\section{Protein production and purification}

Plasmids encoding the heavy and light chains of 1-103, 1-32, 2-18, 2-25 and 8I21 IgG with an $\mathrm{HRV} 3 \mathrm{C}$ protease cleavage site engineered into the hinge between the $\mathrm{CH} 1$ and $\mathrm{CH} 2$ domains of the heavy chain were co-transfected into FreeStyle 293F cells using polyethylenimine. To produce the soluble ectodomain of the HCMV Pentamer (strain Towne), plasmids encoding residues $24-718$ of $\mathrm{gH}$ with a C-terminal 6x HisTag, residues 31-278 of gL, residues 21-171 of UL128, residues 26-214 of UL130 and residues 19-129 of UL131, all with artificial signal sequences were simultaneously co-transfected at an equimolar ratio.

Similarly, plasmids encoding an artificial signal peptide, residues 23-595 of human NRP2 and a C-terminal HRV3C cleavage site with either an 8x HisTag and a TwinStrepTag or a monomeric IgG1 Fc tag and an 8x HisTag were transfected into FreeStyle 293F cells, as described above. An N-terminal truncation of NRP2 that encompassed residues 145-595 with an artificial signal sequence and a Cterminal HRV3C cleavage site with a monomeric IgG1 Fc tag and an 8x HisTag (NRP2 a2b1b2) was transfected using the same conditions. NRP2 and NRP2 a2b1b2 were purified from cell supernatants using either StrepTactin resin (IBA) or Protein A resin before being run over a Superdex 200 Increase column using a buffer composed of $2 \mathrm{mM}$ Tris $\mathrm{pH} 8.0,200 \mathrm{mM} \mathrm{NaCl}, 0.02 \% \mathrm{NaN}_{3}$ and $2 \mathrm{mM} \mathrm{CaCl}$.

To form the complex of Pentamer $+1-103+1-32+2-25$, purified 1-103 IgG was immobilized to Protein A resin and this 1-103 resin was then used to capture Pentamer from co-transfected cell supernatants. The 1-103 + Pentamer complex was then eluted by incubation with HRV3C protease and purified over a Superose 6 Increase column in $2 \mathrm{mM}$ Tris $\mathrm{pH} 8.0,200 \mathrm{mM} \mathrm{NaCl}$ and $0.02 \% \mathrm{NaN}_{3}$. This complex was then passed over a column containing 2-25 IgG immobilized to Protein A resin. Again, the complex was eluted by incubation with HRV3C protease and a molar excess of 1-32 Fab was added before a final round of purification over a Superose 6 Increase column using the same buffer.

To form the Pentamer $+2-18+8 I 21$ complex, purified 2-18 IgG was immobilized to Protein A resin and used to capture Pentamer from co-transfected cell supernatants. The 2-18 + Pentamer complex was then eluted by incubation with HRV3C protease and mixed with a molar excess of 8121 Fab before being run over a Superose 6 Increase column in $2 \mathrm{mM}$ Tris $\mathrm{pH} 8.0,200 \mathrm{mM} \mathrm{NaCl}$ and $0.02 \% \mathrm{NaN}_{3}$. 
To form the Pentamer + NRP2 complex, purified Pentamer was mixed with a threefold molar excess of $8 x$ His/TwinStrep-tagged NRP2 in a buffer composed of $2 \mathrm{mM}$ Tris $\mathrm{pH}$ 8.0, $200 \mathrm{mM} \mathrm{NaCl}$, $0.02 \% \mathrm{NaN}_{3}$ and $2 \mathrm{mM} \mathrm{CaCl}_{2}$ and the two components were allowed to bind on ice for 1 hour. This mixture was then purified over a Superose 6 Increase column (Cytiva) using the same buffer.

X-ray crystallographic studies

Purified IgGs 1-103, 1-32, 2-18 and 2-25 were incubated with 10\% (wt/wt) His-tagged HRV3C protease on ice for 2 hours before being passed over Protein A and NiNTA resin to removed cleaved Fc

312 and excess protease. The remaining Fab was purified by SEC using a Superdex 200 Increase column in $3132 \mathrm{mM}$ Tris $\mathrm{pH}$ 8.0, $200 \mathrm{mM} \mathrm{NaCl}$ and $0.02 \% \mathrm{NaN}_{3}(1-132$ and 2-18) or $2 \mathrm{mM}$ Tris $\mathrm{pH} 8.0,50 \mathrm{mM} \mathrm{NaCl}$ 314 and $0.02 \% \mathrm{NaN}_{3}(1-103$ and $2-25)$.

315 1-103 Fab was concentrated to $15.00 \mathrm{mg} / \mathrm{mL}$ and used to prepare sitting-drop crystallization 316 trays. Diffraction-quality crystals grew in a mother liquor composed of $2.1 \mathrm{M}$ sodium formate, $25 \%$ PEG $3173350,0.1 \mathrm{M}$ sodium acetate $\mathrm{pH} 4.5$ and $0.1 \mathrm{M}$ calcium chloride. 1-103 Fab crystals were cryoprotected 318 using mother liquor supplemented with $20 \%$ glycerol before being plunge frozen into liquid nitrogen. 1-32 Fab was concentrated to $11.00 \mathrm{mg} / \mathrm{mL}$ and used to prepare hanging-drop crystallization

321 isopropanol. 1-32 Fab crystals were cryoprotected using mother liquor supplemented with $20 \%$ glycerol 322 before being plunge frozen into liquid nitrogen.

$3232-18$ Fab was concentrated to $12.00 \mathrm{mg} / \mathrm{mL}$ and used to prepare sitting-drop crystallization trays.

324 Small crystalline needles, grown in 0.1 M HEPES pH 7.5 and 45\% PEG 400 were used to perform

325 microseed matrix screening, ultimately yielding diffraction-quality crystals in a mother liquor composed of $3260.2 \mathrm{M}$ ammonium acetate, $0.1 \mathrm{M}$ sodium citrate tribasic dihydrate $\mathrm{pH} 5.6$ and 30\% PEG 4000. 2-18 Fab 327 crystals were cryoprotected using mother liquor supplemented with $20 \%$ glycerol before being plunge 328 frozen into liquid nitrogen.

$3292-25 \mathrm{Fab}$ was concentrated to $15.4 \mathrm{mg} / \mathrm{mL}$ and used to prepare sitting-drop crystallization trays. 330 Diffraction-quality crystals were grown in $30 \%$ PEG 4000, a mixture of $0.2 \mathrm{M}$ divalent cations ${ }^{51}$ and $0.1 \mathrm{M}$ 
331 BIS-TRIS pH 6.5. 2-25 Fab crystals were looped without cryoprotectant and directly plunge frozen into liquid nitrogen.

All diffraction data were collected at Argonne National Labs, Advanced Photon Source, SBC19ID. Datasets were indexed in iMOSFLM ${ }^{52}$ and scaled in AIMLESS ${ }^{53}$. Molecular replacement solutions were determined using PhaserMR ${ }^{54}$ and models were iteratively built and refined using Coot ${ }^{55}$, PHENIX $^{56}$ and ISOLDE ${ }^{57}$. Full crystallographic data collection and refinement statistics can be found in

337 Supplementary Table 1. Crystallographic software packages were curated by SBGrid 58 .

Cryo-EM sample preparation and data collection attempts to visualize the Pentamer $+2-18$ complex were hampered by a lack of distinguishable features

343 (data not shown). $3 \mu \mathrm{L}$ of the ternary complex was deposited on a CF-1.2/1.3 grid that was glow discharged 344 at $25 \mathrm{~mA}$ for 1 minute using an Emitech K100X (Quorum Technologies). Excess liquid was blotted away 345 for 6 seconds in a Vitrobot Mark IV (FEI) operating at $4^{\circ} \mathrm{C}$ and $100 \%$ humidity before being plunge frozen 346 into liquid ethane. Data were collected on a Titan Krios (FEI) operating at $300 \mathrm{kV}$, equipped with a K3 direct 347 electron detector (Gatan). Movies were collected using SerialEM ${ }^{59}$ at 22,500x magnification, corresponding 348 to a pixel size of $1.047 \AA$.

Purified HCMV Pentamer + 1-103 + 1-32 + 2-25 complex was diluted to a concentration of 0.2

$350 \mathrm{mg} / \mathrm{mL}$ in $2 \mathrm{mM}$ Tris $\mathrm{pH} 8.0,400 \mathrm{mM} \mathrm{NaCl}, 0.02 \% \mathrm{NaN}_{3}$ and $0.01 \%$ amphipol A8-35. $3 \mu \mathrm{L}$ of protein was

351 deposited on a CF-1.2/1.3 grid that was plasma cleaned at $25 \mathrm{~mA}$ for 30 seconds using a Solarus plasma 352 cleaner (Gatan). Excess liquid was blotted away for 6 seconds in a Vitrobot Mark IV (FEI) operating at $4^{\circ}$ $353 \mathrm{C}$ and $100 \%$ humidity before being plunge frozen into liquid ethane. Data were collected on a Titan Krios 354 (FEI) operating at $300 \mathrm{kV}$, equipped with a K2 direct electron detector (Gatan). Movies were collected using 355 Leginon ${ }^{60}$ at $22,500 x$ magnification, corresponding to a pixel size of $1.075 \AA$. 
plasma cleaner (Gatan). Excess liquid was blotted away for 3 seconds in a Vitrobot Mark IV (FEI) operating at $4^{\circ} \mathrm{C}$ and $100 \%$ humidity before being plunge frozen into liquid ethane. Data were collected on a Titan Krios (FEI) operating at $300 \mathrm{kV}$, equipped with a K3 direct electron detector (Gatan). Movies were collected using SerialEM ${ }^{59}$ at $22,500 x$ magnification, corresponding to a pixel size of $1.073 \AA$.

Cryo-EM data processing and model building

Motion correction, CTF-estimation and non-templated particle picking using BoxNet were performed in Warp ${ }^{61}$. Extracted particles were imported into cryoSPARC ${ }^{62}$ for 2D classification, ab initio 3D reconstruction calculation, 3D classification and non-uniform refinement ${ }^{63}$. Based on the flexibility of

368 the interface between the $\mathrm{gH} / \mathrm{gL}$ and UL proteins, particle subtraction and focused refinement were also performed in cryoSPARC. Final reconstructions were sharpened with DeepEMhancer ${ }^{64}$. A full description of the cryo-EM data processing workflows can be found in Supplementary Figs. 2, 6 and 7. Crystal

371 structures were docked into cryo-EM density maps using Chimera ${ }^{65}$ before being refined in Coot $^{55}$,

372 PHENIX ${ }^{56}$ and ISOLDE ${ }^{57}$. A detailed description of the cryo-EM data processing workflow can be found in

373 Supplementary Figs. 2, 6 and 7. Full cryo-EM data collection and refinement statistics can be found in

\section{Supplementary Table 2.}

Surface plasmon resonance (SPR)

377 Purified His-tagged Pentamer was immobilized to a single flow cell of a NiNTA sensor in a

378 Biacore X100 (GE Healthcare) to a level of $\sim 800$ response units (RUs) using HBS-P+ buffer adjusted to a $379 \mathrm{pH}$ of 8.0. Two-fold serial dilutions of Fabs 1-103, 1-32, 2-18 and 2-25 were injected over both flow cells 380 to measure binding kinetics. The sensor was doubly regenerated using $350 \mathrm{mM}$ EDTA and $100 \mathrm{mM}$

$381 \mathrm{NaOH}$ in between cycles. Data were double reference-subtracted and fit to a 1:1 binding model using

382 Biacore Evaluation Software (GE Healthcare). 
BSA and $2 \mathrm{mM} \mathrm{CaCl}_{2}$. Sensors were then dipped into wells containing purified HCMV Pentamer, ranging in concentration from $50 \mathrm{nM}$ to $3.125 \mathrm{nM}$. Data were reference subtracted and processed using Octet Data Analysis software v10.0 (ForteBio) with a 1:1 binding model. To evaluate the impact of calcium on Pentamer binding, the same experiment was performed using monoFc-tagged NRP2 in a buffer composed of $10 \mathrm{mM}$ HEPES pH 8.0, $150 \mathrm{mM} \mathrm{NaCl}, 0.05 \%$ Tween 20, $1 \mathrm{mg} / \mathrm{mL}$ BSA and $2 \mathrm{mM}$ EDTA. software v10.0.

To measure the binding kinetics of NRP2 to Pentamer in the presence of mAb 1-32, 1-32 lgG was immobilized to AHC sensors using a buffer composed of $10 \mathrm{mM} \mathrm{HEPES} \mathrm{pH} \mathrm{8.0,150} \mathrm{mM} \mathrm{NaCl,}$ $0.05 \%$ Tween $20,1 \mathrm{mg} / \mathrm{mL}$ BSA and $2 \mathrm{mM} \mathrm{CaCl} 2$. Tips with immobilized 1-32 were then dipped into wells containing $100 \mathrm{nM}$ Pentamer. The 1-32-captured Pentamer was then dipped into wells containing untagged NRP2, ranging in concentration from $400 \mathrm{nM}$ to $25 \mathrm{nM}$. Data were reference subtracted and

HCMV neutralization assay processed using Octet Data Analysis software v10.0 with a 1:1 binding model.

407 isotype control. Fabs for neutralization and inhibition assays were generated by digesting IgG with papain 408 (Sigma, P4762) and purifying as described previously67. A standard neutralization assay with the Towne409 ts15-rR, AD169rev, and 12 clinical isolates as shown in Supplementary Fig. 10 were performed in ARPE41019 cells using an immunostaining method ${ }^{68}$. Neutralization assays in Fig. 4 were performed in ARPE-19 411 cells using AD169rev-GFP strain and virus infection was examined through GFP expression as described 412 previously ${ }^{69}$. For the standard neutralization assay, $50 \mu \mathrm{L} /$ well of AD169rev-GFP, generating about 100 413 GFP-positive cells was incubated with $50 \mu \mathrm{L} /$ well of serial 2-fold diluted IgG or Fab (at indicated 414 concentrations) at $37^{\circ} \mathrm{C}$ for 30 min and then added to confluent ARPE-19 cells grown in a 96-well plate. 
415 Mock-infected cells and cells infected with virus-only served as controls. For the post-attachment assay,

416 ARPE-19 cells grown in a 96-well plate were pre-cooled at $4{ }^{\circ} \mathrm{C}$ for $10 \mathrm{~min} .50 \mu \mathrm{L} /$ well of AD169rev-GFP

417 was allowed to attach to cells for $1 \mathrm{~h}$ at $4{ }^{\circ} \mathrm{C}$. After removing unattached virus through a single wash using

418 cold media, the indicated IgG, diluted at concentrations of $\sim 200$ times of corresponding IC 50 was added

419 after culturing AD169rev-GFP-attached cells for different lengths of time (0 min, $30 \mathrm{~min}$, and $60 \mathrm{~min})$ in a

$42037^{\circ} \mathrm{C}$ incubator. The antibody-containing media was replaced with fresh media without antibody $2 \mathrm{~h}$ later.

421 Mock-infected cells and cells infected with virus but not treated with antibodies served as controls. For all

422 above assays, triplicate wells were determined for each condition and viral infection was examined at $48 \mathrm{~h}$

423 post-infection. A C.T.L. Immunospot analyzer was used to capture whole-well images of GFP expression

424 and quantification of GFP-positive cells. The percentage of viral inhibition by the antibody and the IC50 of

425 each antibody was calculated by non-linear fit of virus inhibition \% vs. concentration ( $\mathrm{ng} / \mathrm{mL})$ using

\section{GraphPad Prism® 5 software.}




\section{REFERENCES}

1 Gerna, G. et al. Dendritic-cell infection by human cytomegalovirus is restricted to strains carrying functional UL131-128 genes and mediates efficient viral antigen presentation to CD8+ T cells. J Gen Virol 86, 275-284, doi:10.1099/vir.0.80474-0 (2005).

2 Vanarsdall, A. L., Chase, M. C. \& Johnson, D. C. Human cytomegalovirus glycoprotein gO complexes with $\mathrm{gH} / \mathrm{gL}$, promoting interference with viral entry into human fibroblasts but not entry into epithelial cells. J Virol 85, 11638-11645, doi:10.1128/JVI.05659-11 (2011).

3 Adler, B. et al. Role of human cytomegalovirus UL131A in cell type-specific virus entry and release. J Gen Virol 87, 2451-2460, doi:10.1099/vir.0.81921-0 (2006).

4 Martinez-Martin, N. et al. An Unbiased Screen for Human Cytomegalovirus Identifies Neuropilin-2 as a Central Viral Receptor. Cell 174, 1158-1171 e1119, doi:10.1016/j.cell.2018.06.028 (2018).

5 Teesalu, T., Sugahara, K. N., Kotamraju, V. R. \& Ruoslahti, E. C-end rule peptides mediate neuropilin-1-dependent cell, vascular, and tissue penetration. Proc Natl Acad Sci U S A 106, 16157-16162, doi:10.1073/pnas.0908201106 (2009).

6 Tsai, Y. C. et al. Structural studies of neuropilin-2 reveal a zinc ion binding site remote from the vascular endothelial growth factor binding pocket. FEBS J 283, 1921-1934, doi:10.1111/febs.13711(2016).

7 Cannon, M. J., Schmid, D. S. \& Hyde, T. B. Review of cytomegalovirus seroprevalence and demographic characteristics associated with infection. Rev Med Virol 20, 202-213, doi:10.1002/rmv.655 (2010).

8 Ahlfors, K., Forsgren, M., Ivarsson, S. A., Harris, S. \& Svanberg, L. Congenital cytomegalovirus infection: on the relation between type and time of maternal infection and infant's symptoms. Scand J Infect Dis 15, 129-138, doi:10.3109/inf.1983.15.issue2.01 (1983).

9 Pass, R. F., Fowler, K. B., Boppana, S. B., Britt, W. J. \& Stagno, S. Congenital cytomegalovirus infection following first trimester maternal infection: symptoms at birth and outcome. J Clin Virol 35, 216-220, doi:10.1016/j.jcv.2005.09.015 (2006).

10 Roark, H. K., Jenks, J. A., Permar, S. R. \& Schleiss, M. R. Animal Models of Congenital Cytomegalovirus Transmission: Implications for Vaccine Development. J Infect Dis 221, S60-S73, doi:10.1093/infdis/jiz484 (2020).

11 Ligat, G., Cazal, R., Hantz, S. \& Alain, S. The human cytomegalovirus terminase complex as an antiviral target: a close-up view. FEMS Microbiol Rev 42, 137-145, doi:10.1093/femsre/fuy004 (2018).

12 Mumtaz, K. et al. Universal prophylaxis or preemptive strategy for cytomegalovirus disease after liver transplantation: a systematic review and meta-analysis. Am J Transplant 15, 472-481, doi:10.1111/ajt.13044 (2015).

13 McGeoch, D. J., Cook, S., Dolan, A., Jamieson, F. E. \& Telford, E. A. Molecular phylogeny and evolutionary timescale for the family of mammalian herpesviruses. J Mol Biol 247, 443-458, doi:10.1006/jmbi.1995.0152 (1995). 
47014 Isaacson, M. K. \& Compton, T. Human cytomegalovirus glycoprotein B is required for

471

472

473

474

475

476

477

478

479

480

481

482

483

484

485

486

487

488

489

490

491

492

493

494

495

496

497

498

499

500

501

502

503

504

505

506

507

508

509

510

511

$512 \quad 28$

513 virus entry and cell-to-cell spread but not for virion attachment, assembly, or egress. J Virol 83, 3891-3903, doi:10.1128/JVI.01251-08 (2009).

15 Wang, D. \& Shenk, T. Human cytomegalovirus virion protein complex required for epithelial and endothelial cell tropism. Proc Natl Acad Sci U S A 102, 18153-18158, doi:10.1073/pnas.0509201102 (2005).

16 Zhou, M., Lanchy, J. M. \& Ryckman, B. J. Human Cytomegalovirus gH/gL/gO Promotes the Fusion Step of Entry into All Cell Types, whereas gH/gL/UL128-131 Broadens Virus Tropism through a Distinct Mechanism. J Virol 89, 8999-9009, doi:10.1128/JVI.01325-15 (2015).

17 Ciferri, C. et al. Structural and biochemical studies of HCMV gH/gL/gO and Pentamer reveal mutually exclusive cell entry complexes. Proc Natl Acad Sci U S A 112, 1767-1772, doi:10.1073/pnas.1424818112 (2015).

18 Kabanova, A. et al. Platelet-derived growth factor-alpha receptor is the cellular receptor for human cytomegalovirus gHgLgO trimer. Nat Microbiol 1, 16082, doi:10.1038/nmicrobiol.2016.82 (2016).

19 Wu, Y. et al. Human cytomegalovirus glycoprotein complex gH/gL/gO uses PDGFR-alpha as a key for entry. PLoS Pathog 13, e1006281, doi:10.1371/journal.ppat.1006281 (2017).

20 Kschonsak, M. et al. Structures of HCMV Trimer reveal the basis for receptor recognition and cell entry. Cell, doi:10.1016/j.cell.2021.01.036 (2021).

21 Hahn, G. et al. Human cytomegalovirus UL131-128 genes are indispensable for virus growth in endothelial cells and virus transfer to leukocytes. J Virol 78, 10023-10033, doi:10.1128/JVI.78.18.10023-10033.2004 (2004).

22 Ryckman, B. J. et al. Characterization of the human cytomegalovirus gH/gL/UL128-131 complex that mediates entry into epithelial and endothelial cells. J Virol 82, 60-70, doi:10.1128/JVI.01910-07 (2008).

23 Wille, P. T., Wisner, T. W., Ryckman, B. \& Johnson, D. C. Human cytomegalovirus (HCMV) glycoprotein $\mathrm{gB}$ promotes virus entry in trans acting as the viral fusion protein rather than as a receptor-binding protein. mBio 4, e00332-00313, doi:10.1128/mBio.00332-13 (2013).

24 Soker, S., Takashima, S., Miao, H. Q., Neufeld, G. \& Klagsbrun, M. Neuropilin-1 is expressed by endothelial and tumor cells as an isoform-specific receptor for vascular endothelial growth factor. Cell 92, 735-745, doi:10.1016/s0092-8674(00)81402-6 (1998).

25 Takagi, S., Tsuji, T., Amagai, T., Takamatsu, T. \& Fujisawa, H. Specific cell surface labels in the visual centers of Xenopus laevis tadpole identified using monoclonal antibodies. Dev Biol 122, 90-100, doi:10.1016/0012-1606(87)90335-6 (1987).

26 Dallas, N. A. et al. Neuropilin-2-mediated tumor growth and angiogenesis in pancreatic adenocarcinoma. Clin Cancer Res 14, 8052-8060, doi:10.1158/1078-0432.CCR-08-1520 (2008).

27 Kitsukawa, T., Shimono, A., Kawakami, A., Kondoh, H. \& Fujisawa, H. Overexpression of a membrane protein, neuropilin, in chimeric mice causes anomalies in the cardiovascular system, nervous system and limbs. Development 121, 4309-4318 (1995).

28 Prahst, C. et al. Neuropilin-1-VEGFR-2 complexing requires the PDZ-binding domain of neuropilin-1. J Biol Chem 283, 25110-25114, doi:10.1074/jbc.C800137200 (2008). 
51429 Takagi, S. et al. The A5 antigen, a candidate for the neuronal recognition molecule, has 515 homologies to complement components and coagulation factors. Neuron 7, 295-307, 516 doi:10.1016/0896-6273(91)90268-5 (1991).

$51730 \quad$ Favier, B. et al. Neuropilin-2 interacts with VEGFR-2 and VEGFR-3 and promotes human 518 endothelial cell survival and migration. Blood 108, 1243-1250, doi:10.1182/blood-2005519 11-4447 (2006).

$52031 \quad$ Parker, M. W., Xu, P., Li, X. \& Vander Kooi, C. W. Structural basis for selective vascular Cytomegalovirus into Epithelial and Endothelial Cells. mBio 9, doi:10.1128/mBio.0078118 (2018).

$35 \mathrm{Ha}, \mathrm{S}$. et al. Neutralization of Diverse Human Cytomegalovirus Strains Conferred by Antibodies Targeting Viral gH/gL/pUL128-131 Pentameric Complex. J Virol 91, doi:10.1128/JVI.02033-16 (2017).

Vanarsdall, A. L. et al. HCMV trimer- and pentamer-specific antibodies synergize for virus neutralization but do not correlate with congenital transmission. Proc Natl Acad Sci U S A 116, 3728-3733, doi:10.1073/pnas.1814835116 (2019).

37 Freed, D. C. et al. Pentameric complex of viral glycoprotein $\mathrm{H}$ is the primary target for potent neutralization by a human cytomegalovirus vaccine. Proc Natl Acad Sci U S A 110, E4997-5005, doi:10.1073/pnas.1316517110 (2013).

Appleton, B. A. et al. Structural studies of neuropilin/antibody complexes provide insights into semaphorin and VEGF binding. EMBO J 26, 4902-4912, doi:10.1038/sj.emboj.7601906 (2007). Lujo virus. Nat Microbiol 3, 1153-1160, doi:10.1038/s41564-018-0224-5 (2018). Parker, M. W. et al. Structural basis for VEGF-C binding to neuropilin-2 and sequestration by a soluble splice form. Structure 23, 677-687, doi:10.1016/j.str.2015.01.018 (2015). domains. Proc Natl Acad Sci U S A 104, 6152-6157, doi:10.1073/pnas.0700043104 (2007). human cytomegalovirus. Sci Immunol 2, doi:10.1126/sciimmunol.aan1457 (2017).

43 Xia, L. et al. Active evolution of memory B-cells specific to viral gH/gL/pUL128/130/131 pentameric complex in healthy subjects with silent human cytomegalovirus infection. Oncotarget 8, 73654-73669, doi:10.18632/oncotarget.18359 (2017). 
55644 Ye, X. et al. Recognition of a highly conserved glycoprotein B epitope by a bivalent antibody neutralizing HCMV at a post-attachment step. PLoS Pathog 16, e1008736, doi:10.1371/journal.ppat.1008736 (2020).

$55945 \quad$ Yelland, T. \& Djordjevic, S. Crystal Structure of the Neuropilin-1 MAM Domain: Completing the Neuropilin-1 Ectodomain Picture. Structure 24, 2008-2015, doi:10.1016/j.str.2016.08.017 (2016).

46 Sinzger, C. et al. Fibroblasts, epithelial cells, endothelial cells and smooth muscle cells are major targets of human cytomegalovirus infection in lung and gastrointestinal

$47 \mathrm{Si}$, Z. et al. Different functional states of fusion protein gB revealed on human

49 Gerna, G., Percivalle, E., Perez, L., Lanzavecchia, A. \& Lilleri, D. Monoclonal Antibodies to Different Components of the Human Cytomegalovirus (HCMV) Pentamer $\mathrm{gH} / \mathrm{gL} / \mathrm{pUL} 128 \mathrm{~L}$ and Trimer $\mathrm{gH} / \mathrm{gL} / \mathrm{gO}$ as well as Antibodies Elicited during Primary HCMV Infection Prevent Epithelial Cell Syncytium Formation. J Virol 90, 6216-6223, doi:10.1128/JVI.00121-16 (2016). $\mathrm{gH} / \mathrm{gL} / \mathrm{pUL128/130/131} \mathrm{Complex} \mathrm{of} \mathrm{Human} \mathrm{Cytomegalovirus.} \mathrm{Antimicrob} \mathrm{Agents}$ Chemother 65, doi:10.1128/AAC.02422-20 (2021). graphical interface for diffraction-image processing with MOSFLM. Acta Crystallogr $D$ Crystallogr D Biol Crystallogr 69, 1204-1214, doi:10.1107/S0907444913000061 (2013). McCoy, A. J. Solving structures of protein complexes by molecular replacement with Phaser. Acta Crystallogr D Biol Crystallogr 63, 32-41, doi:10.1107/\$0907444906045975 (2007). Crystallogr D Biol Crystallogr 60, 2126-2132, doi:10.1107/S0907444904019158 (2004). Adams, P. D. et al. PHENIX: building new software for automated crystallographic structure determination. Acta Crystallogr D Biol Crystallogr 58, 1948-1954, doi:10.1107/s0907444902016657 (2002).

59858 Morin, A. et al. Collaboration gets the most out of software. Elife 2, e01456, 
60059 Mastronarde, D. N. SerialEM: A Program for Automated Tilt Series Acquisition on Tecnai

601 Microscopes Using Prediction of Specimen Position. Microscopy and Microanalysis,

602 doi:10.1017/S1431927603445911 (2003).

60360 Carragher, B. et al. Leginon: an automated system for acquisition of images from vitreous ice specimens. J Struct Biol 132, 33-45, doi:10.1006/jsbi.2000.4314 (2000).

61 Tegunov, D. \& Cramer, P. Real-time cryo-electron microscopy data preprocessing with Warp. Nat Methods 16, 1146-1152, doi:10.1038/s41592-019-0580-y (2019).

62 Punjani, A., Rubinstein, J. L., Fleet, D. J. \& Brubaker, M. A. cryoSPARC: algorithms for rapid unsupervised cryo-EM structure determination. Nat Methods 14, 290-296, doi:10.1038/nmeth.4169 (2017). improves single-particle cryo-EM reconstruction. Nat Methods 17, 1214-1221, doi:10.1038/s41592-020-00990-8 (2020). post-processing. bioRxiv, doi:https://doi.org/10.1101/2020.06.12.148296 (2020). Pettersen, E. F. et al. UCSF Chimera--a visualization system for exploratory research and analysis. J Comput Chem 25, 1605-1612, doi:10.1002/jcc.20084 (2004). $\mathrm{Li}$, L. et al. Potent neutralizing antibodies elicited by dengue vaccine in rhesus macaque target diverse epitopes. PLoS pathogens 15, e1007716, doi:10.1371/journal.ppat.1007716 (2019).

67 Ye, X. et al. Structural Basis for Recognition of Human Enterovirus 71 by a Bivalent Broadly Neutralizing Monoclonal Antibody. PLoS pathogens 12, e1005454, doi:10.1371/journal.ppat.1005454 (2016).

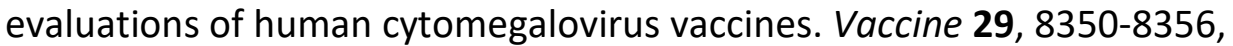
doi:10.1016/j.vaccine.2011.08.086 (2011). 
Figure 1

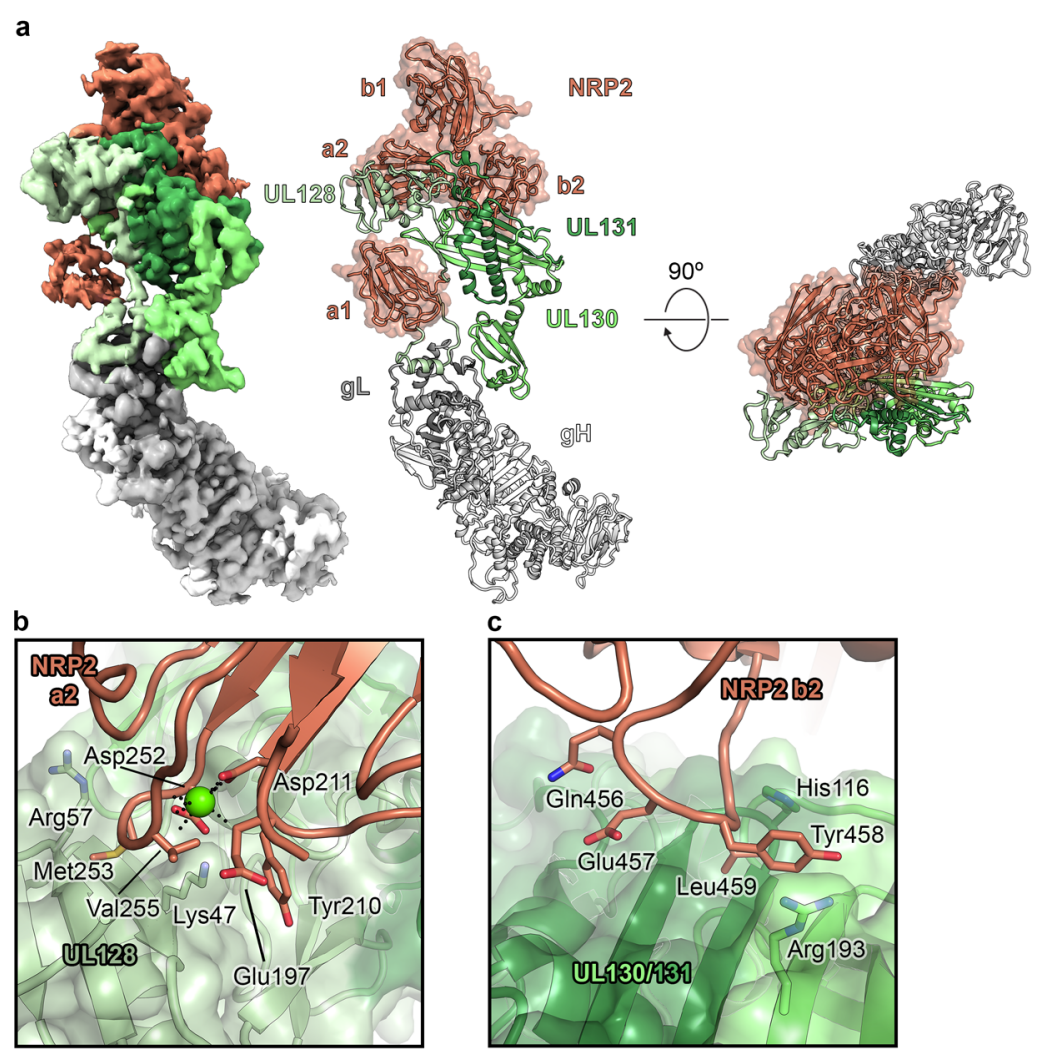

Figure 1: The cryo-EM structure of the HCMV Pentamer bound by NRP2. (a) Cryo-EM density is shown (left), with the Pentamer colored in shades of green, gray and white and NRP2 colored orange. The atomic model of this complex (right) is shown as ribbons, with the NRP2 also represented by a transparent molecular surface. (b) The interface between the NRP2 a2 domain and UL128. UL128 is depicted as a transparent, green molecular surface with ribbons underneath and NRP2 is shown as orange ribbons. Residues that are predicted to form critical contacts are shown as sticks. Oxygen, nitrogen, and sulfur atoms are colored red, blue, and yellow, respectively. The single calcium atom is shown as a bright green sphere, with black dotted lines depicting the interaction with conserved coordinating residues. (c) The interface between the NRP2 b2 domain and the HCMV Pentamer. ULs 130 and 131 are shown as a transparent, green molecular surface and NRP2 is shown as orange ribbons, with residues predicted to form critical contacts shown as sticks. Oxygen and nitrogen atoms are colored red and blue, respectively. 
Figure 2

a

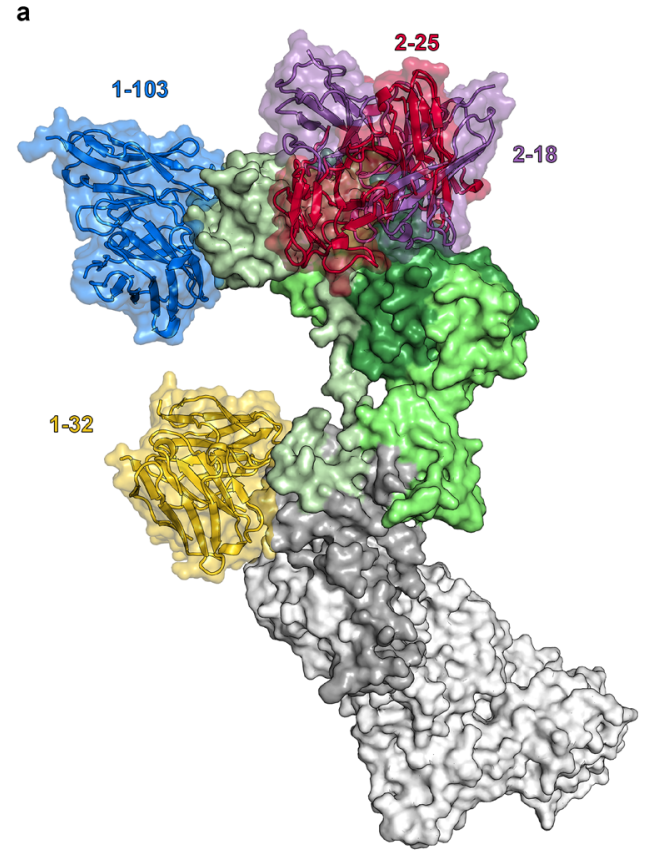

b

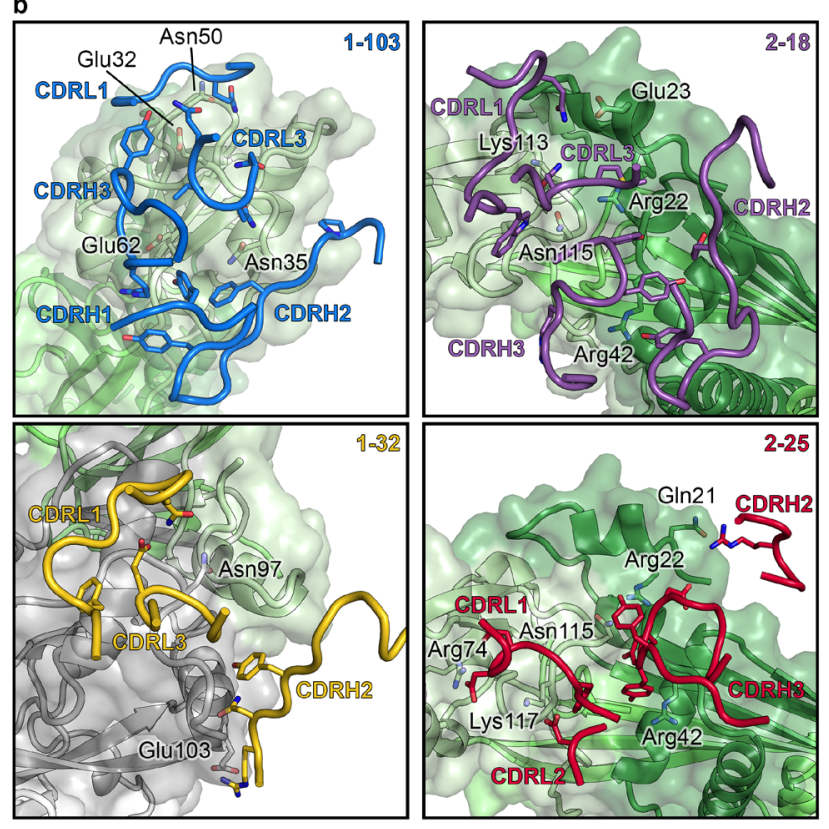

Figure 2: Composite of cryo-EM structures of Pentamer bound by four neutralizing human antibodies. (a) The atomic models of two cryo-EM structures of antibodies bound to the HCMV Pentamer are superimposed based on the position of the UL proteins. The Pentamer is shown as a molecular surface, colored according to Fig. 1 and Fabs are shown as ribbons surrounded by a transparent molecular surface. Fab 1-103 is colored blue, Fab 1-32 is colored gold, Fab 2-18 is colored purple and Fab 2-25 is colored red. (b) CDRs from each Fab are shown as ribbons and the Pentamer is shown as a transparent, molecular surface with ribbons underneath. Predicted critical contact residues are shown as sticks. Fab 1-103 (top left) is colored blue, Fab 1-32 (bottom left) is colored gold, Fab 2-18 (top right) is colored purple and Fab 2-25 (bottom right) is colored red. Oxygen, nitrogen and sulfur atoms are colored red, blue and yellow respectively. 
Figure 3

a
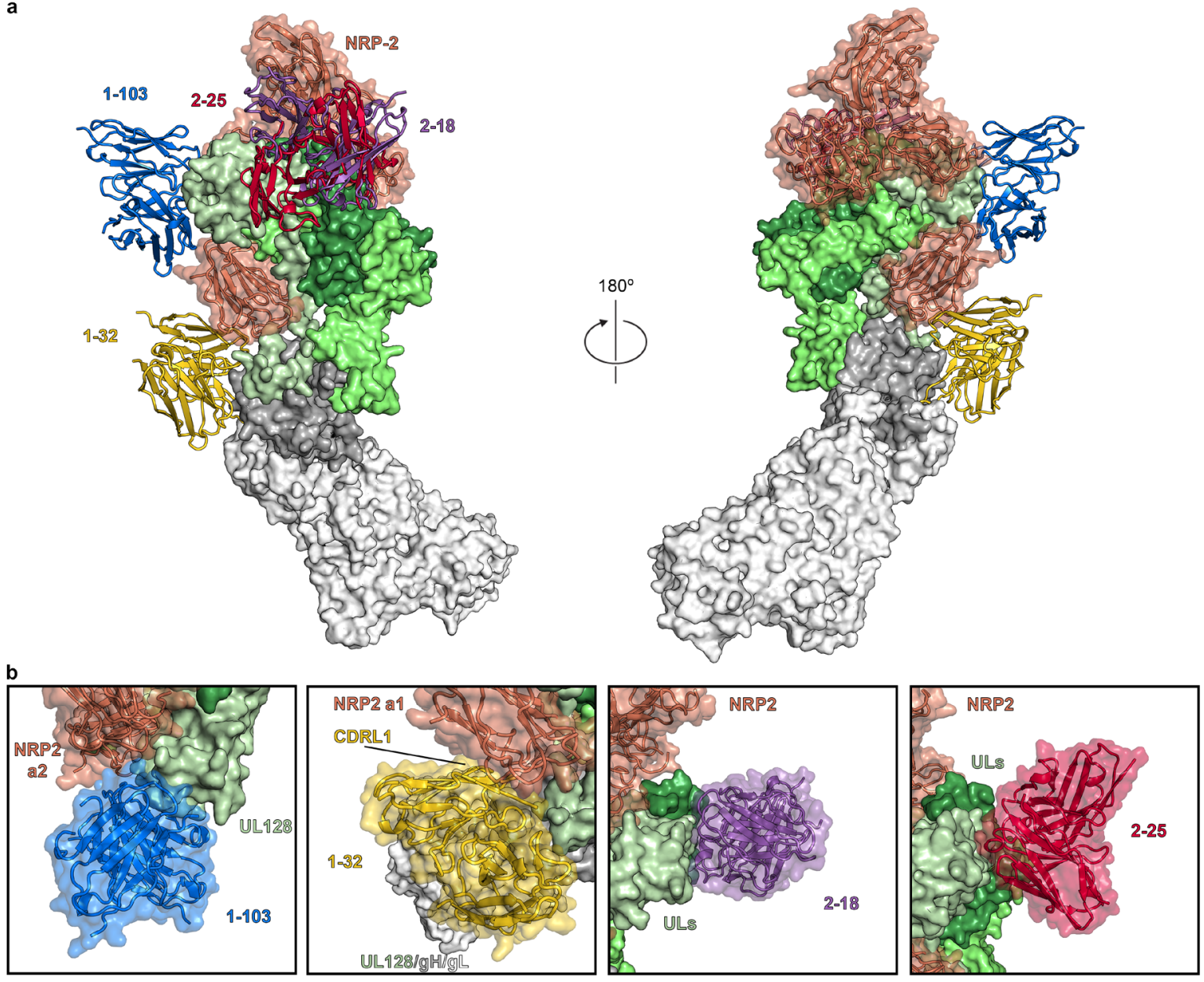

Figure 3: Pentamer-directed antibodies can neutralize HCMV via multiple mechanisms. (a) Cryo-EM structures of NRP2-bound Pentamer and Fab-bound Pentamer are superimposed based on the position of the UL proteins. Pentamer is shown as a molecular surface colored according to Fig. 1, Fabs are shown as ribbons, colored according to Fig. 2, and NRP2 is shown as orange ribbons surrounded by a transparent molecular surface. (b) Close-up views of each Fab-bound Pentamer are superimposed upon the NRP2-bound Pentamer. Both Fabs and NRP2 are shown as ribbons surrounded by a transparent molecular surface, while the Pentamer is shown as a solid molecular surface. NRP2 is colored orange, 1-103 is colored blue, 1-32 is colored gold, 2-18 is colored purple and 2-25 is colored red. 
Figure 4

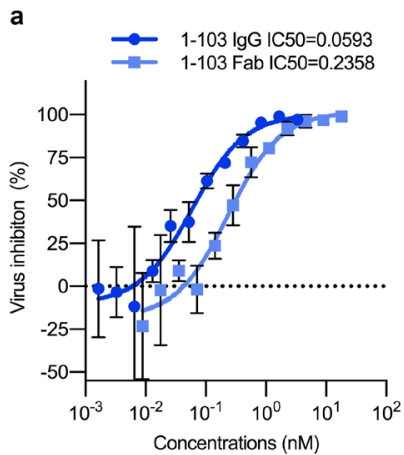

b

HCMV Pentamer binding to NRP-2 with Fabs present

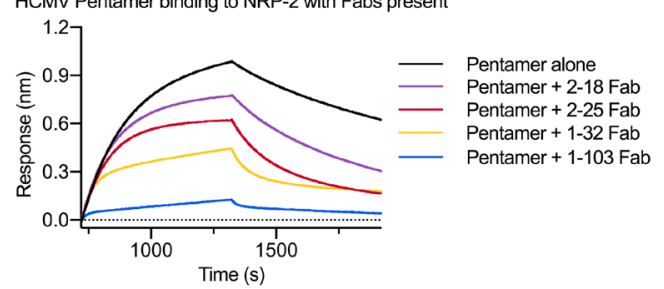

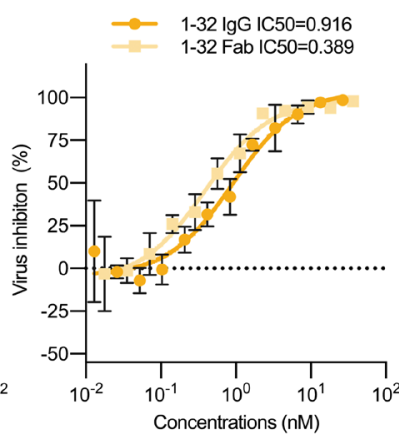

c

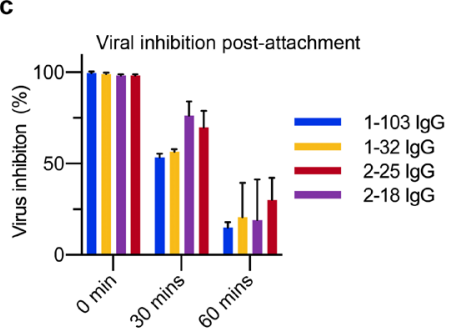

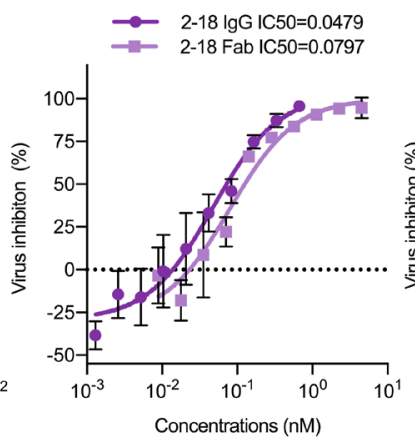

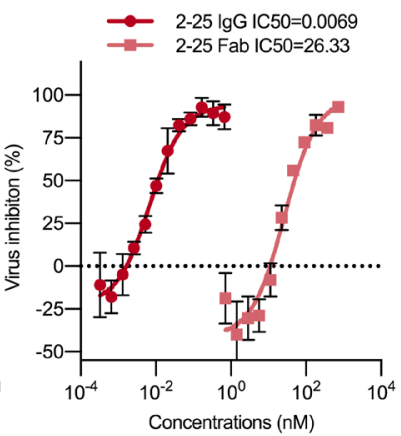

d

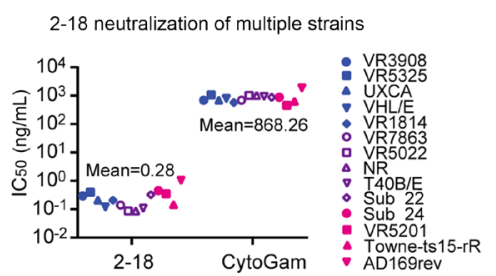

Figure 4: Antibodies 2-18 and 2-25 potently neutralize HCMV without disrupting NRP2 binding. (a) Neutralization curves are shown for each mAb based on inhibition of AD169revGFP infection in ARPE-19 cells. Inhibitory curves for both IgG and Fab are shown, with IgG shown in darker colors. (b) Sensorgrams from a BLI-based competition experiment are displayed. NRP2 was immobilized to a BLI sensor and dipped into Pentamer alone or Pentamer incubated with a molar excess of indicated Fab. (c) IgG neutralization of HCMV post-attachment to epithelial cells. AD169rev-GFP virions were allowed to adhere to ARPE-19 cells and saturating concentrations of IgG were added after a variety of different incubation periods. Viral inhibition is plotted for each IgG after either 0 mins incubation, 30 mins incubation, or 60 mins incubation. (d) Neutralization potency of 2-18 IgG was evaluated against twelve clinical isolates and two laboratory-adapted HCMV strains in ARPE-19 cells. $\mathrm{IC}_{50}$ values were calculated by non-linear fit of the percentage of viral inhibition vs. concentration $(\mathrm{ng} / \mathrm{mL})$. The neutralization results of mAbs 1-103, 1-32 and 2-25 against the same panel of HCMV strains have been reported previously ${ }^{35}$. 


\section{Supplementary Figure 1}

a
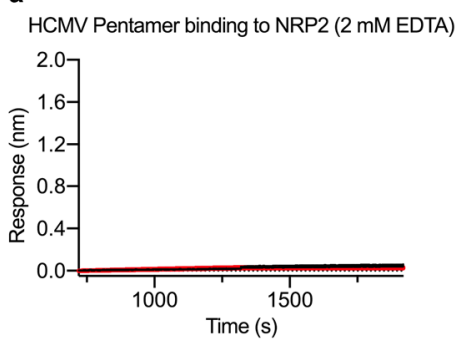

c

HCMV Pentamer binding to NRP2 a2b1b2 (2mM Ca $\left.{ }^{2+}\right)$

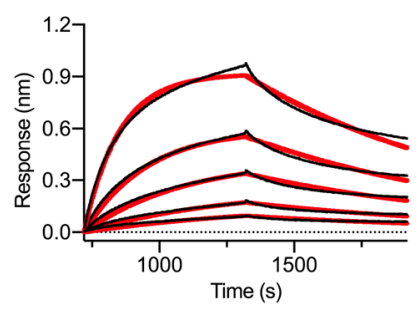

b

HCMV Pentamer binding to NRP2 $\left(2 \mathrm{mM} \mathrm{Ca}^{2+}\right)$

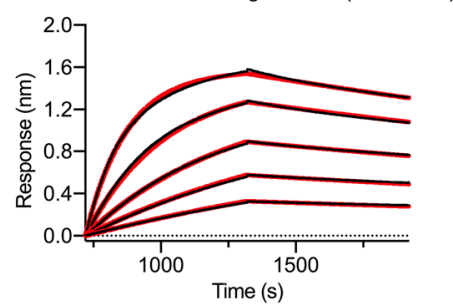

$K_{\mathrm{D}}=2.16 \times 10^{-9} \mathrm{M}$ $k_{\mathrm{a}}=1.25 \times 10^{5} \mathrm{M}^{-1} \mathrm{~s}^{-1}$ $k_{\mathrm{d}}=2.71 \times 10^{-4} \mathrm{~s}^{-1}$

$K_{\mathrm{D}}=7.88 \times 10^{-9} \mathrm{M}$

$k_{\mathrm{a}}=1.32 \times 10^{5} \mathrm{M}^{-1} \mathrm{~s}^{-1}$

$k_{\mathrm{d}}=1.04 \times 10^{-3} \mathrm{~s}^{-1}$

Supplementary Figure 1: The interaction between NRP2 and Pentamer is calcium-dependent. (a) BLI sensorgram showing the absence of binding between Pentamer and NRP2 in the presence of $2 \mathrm{mM}$ EDTA. (b) BLI sensorgram showing binding between Pentamer and NRP2 in the presence of $2 \mathrm{mM}$ calcium. Data are shown as black lines and best fit of a 1:1 binding model is shown as red lines. (c) BLI sensorgram showing binding between Pentamer and NRP2 a2b1b2 in the presence of $2 \mathrm{mM}$ calcium. Data are shown as black lines and best fit of a 1:1 binding model is shown as red lines. 
bioRxiv preprint doi: https://doi.org/10.1101/2021.03.25.436804; this version posted March 25, 2021. The copyright holder for this preprint (which was not certified by peer review) is the author/funder, who has granted bioRxiv a license to display the preprint in perpetuity. It is made available under aCC-BY-NC-ND 4.0 International license.

Supplementary Figure 2

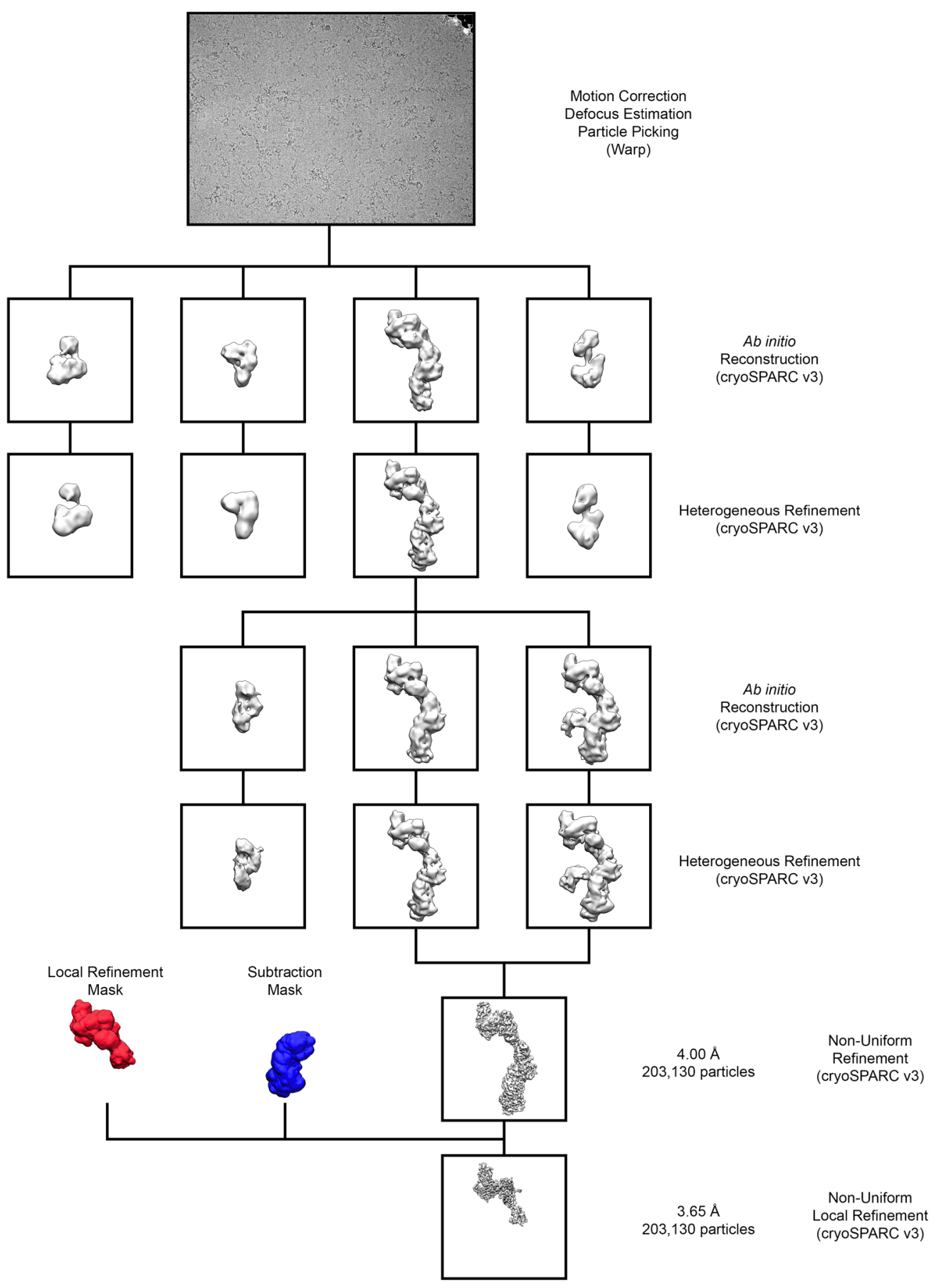

Supplementary Figure 2: Pentamer + NRP2 cryo-EM data processing workflow. 
Supplementary Figure 3
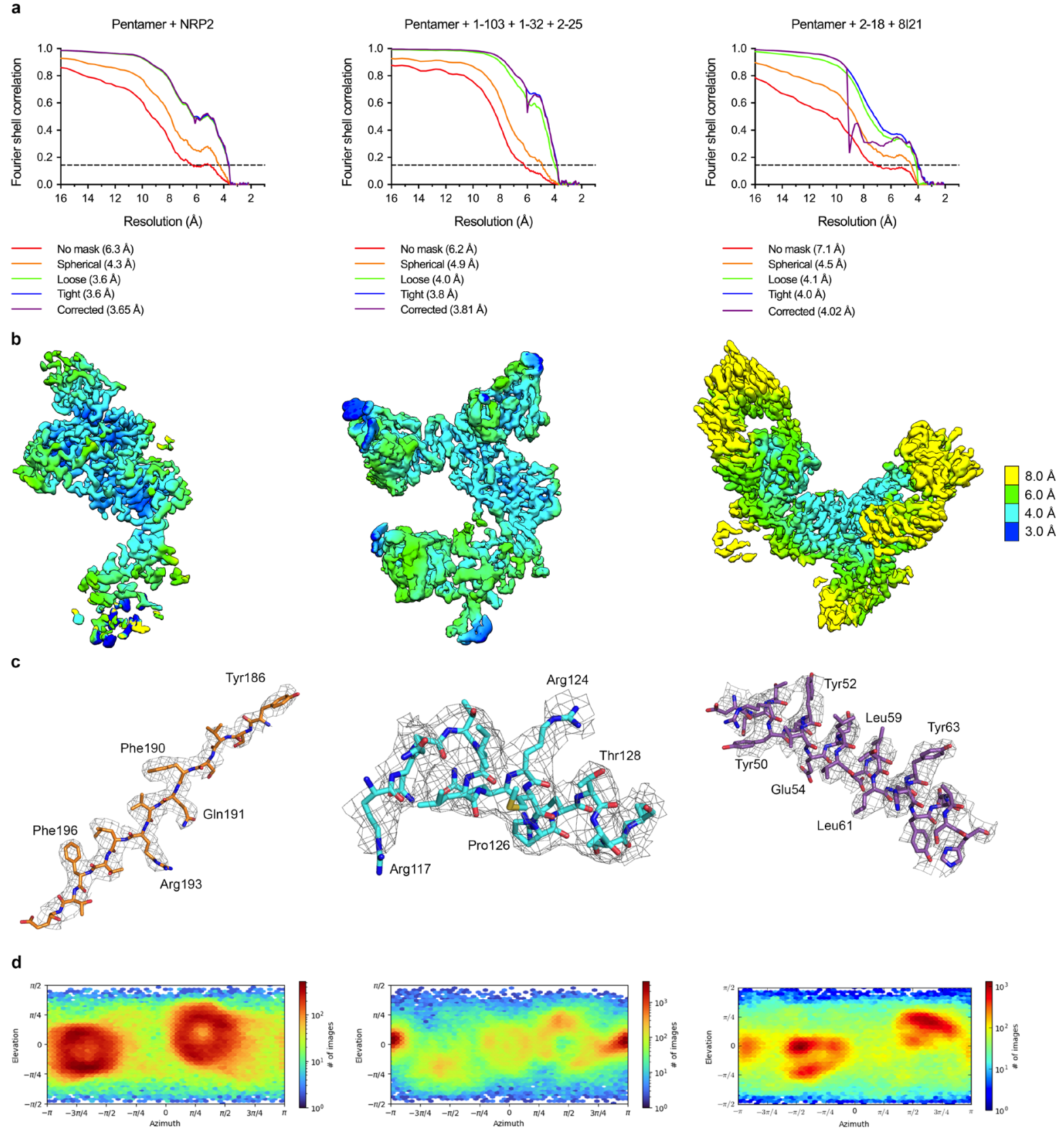

Supplementary Figure 3: Cryo-EM structure validation. (a) FSC curves are shown for focused refinements of Pentamer bound by NRP2 (left), Pentamer bound by 1-103, 1-32 and 2-25 (middle) and Pentamer bound by 2-18 and 8I21 (right). (b) Cryo-EM maps from each focused refinement are shown, colored according to local resolution. (c) Portions of each cryo-EM map are shown, with the corresponding atomic models docked into the density. Residue numbering corresponds to UL130 (left, middle) and UL131 (right). (d) Viewing Direction Distribution charts from cryoSPARC are shown for each focused refinement. 


\section{Supplementary Figure 4}

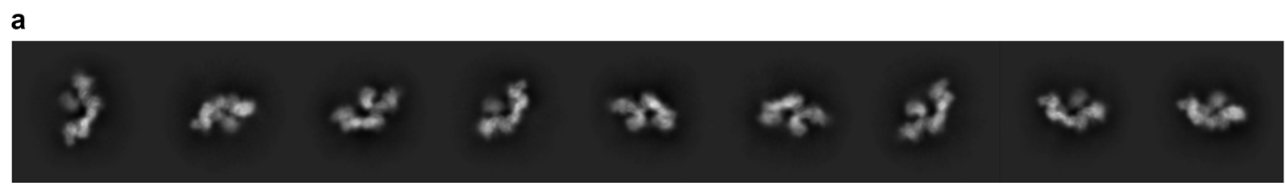

b
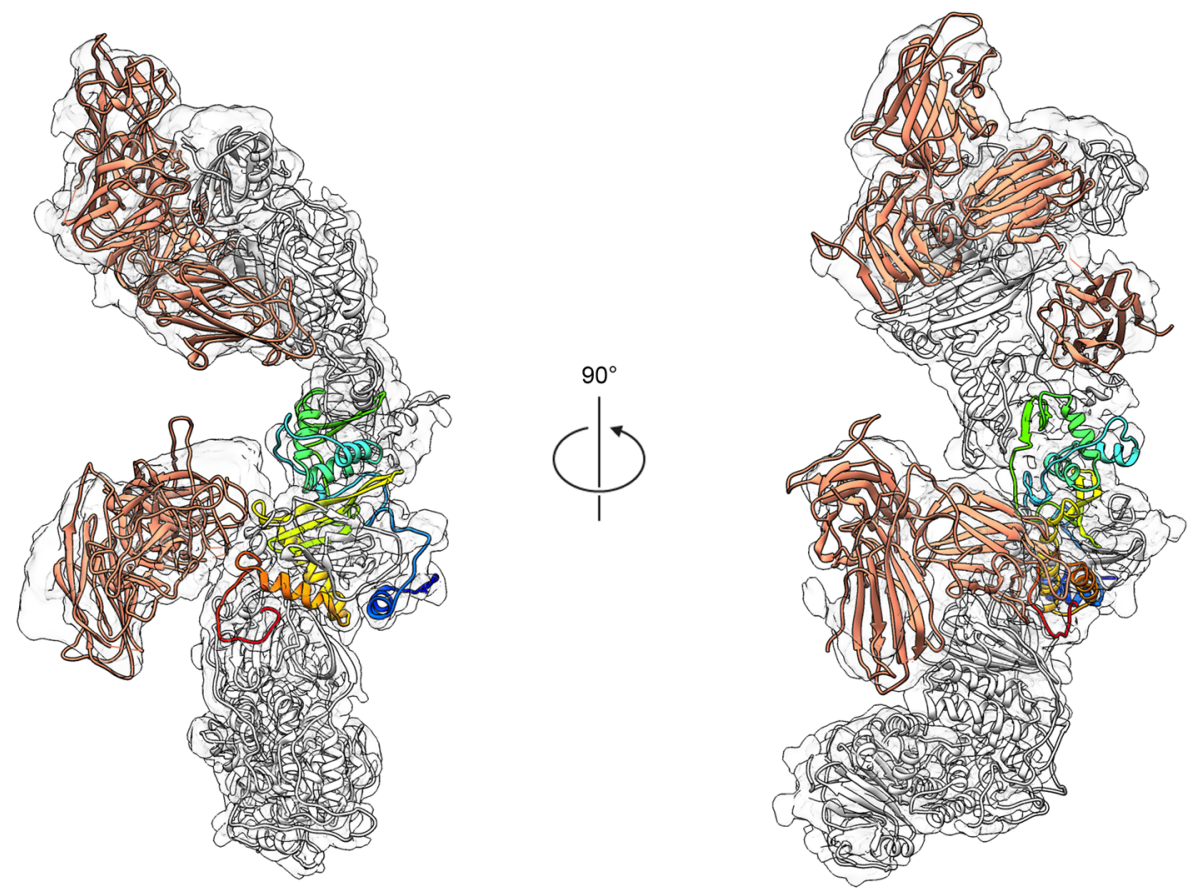

Supplementary Figure 4: A subset of particles display a second copy of NRP2 bound to the Cterminus of gL. (a) Two-dimensional class averages of Pentamer bound by two copies of NRP2 are shown. (b) A $\sim 4.2 \AA$ cryo-EM reconstruction of Pentamer bound by two copies of NRP2 is shown as a transparent surface. Atomic models of each component are docked in, shown as ribbons. Both copies of NRP2 are colored orange and Pentamer is colored white, except for gL, which is colored blue-to-red from the N-terminus to the C-terminus. The al domain from the gLbound copy of NRP2 was excluded because it could not clearly be resolved in this reconstruction. 
Supplementary Figure 5

a

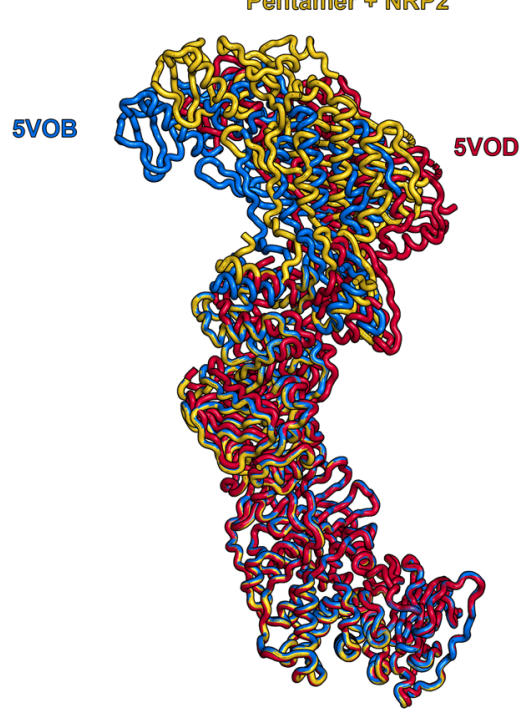

b

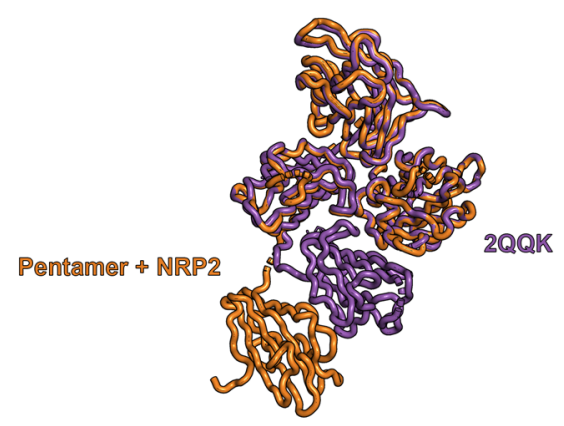

Supplementary Figure 5: NRP2 binding does not alter the conformation of Pentamer. (a) Previously reported crystal structures of Pentamer ${ }^{42}$ (PDB IDs: 5V0B and 5V0D) are aligned to the cryo-EM structure of the NRP2-bound Pentamer, based on the position of gH. 5V0B is colored blue, 5V0D is colored red and NRP2-bound Pentamer is colored yellow. (b) A previously reported crystal structure of NRP2 ${ }^{38}$ (PDB ID: 2QQK) is aligned to the cryo-EM structure of Pentamer-bound NRP2, based on the position of the b1 domain. 2QQK is colored purple and Pentamer-bound NRP2 is colored orange. 
Supplementary Figure 6

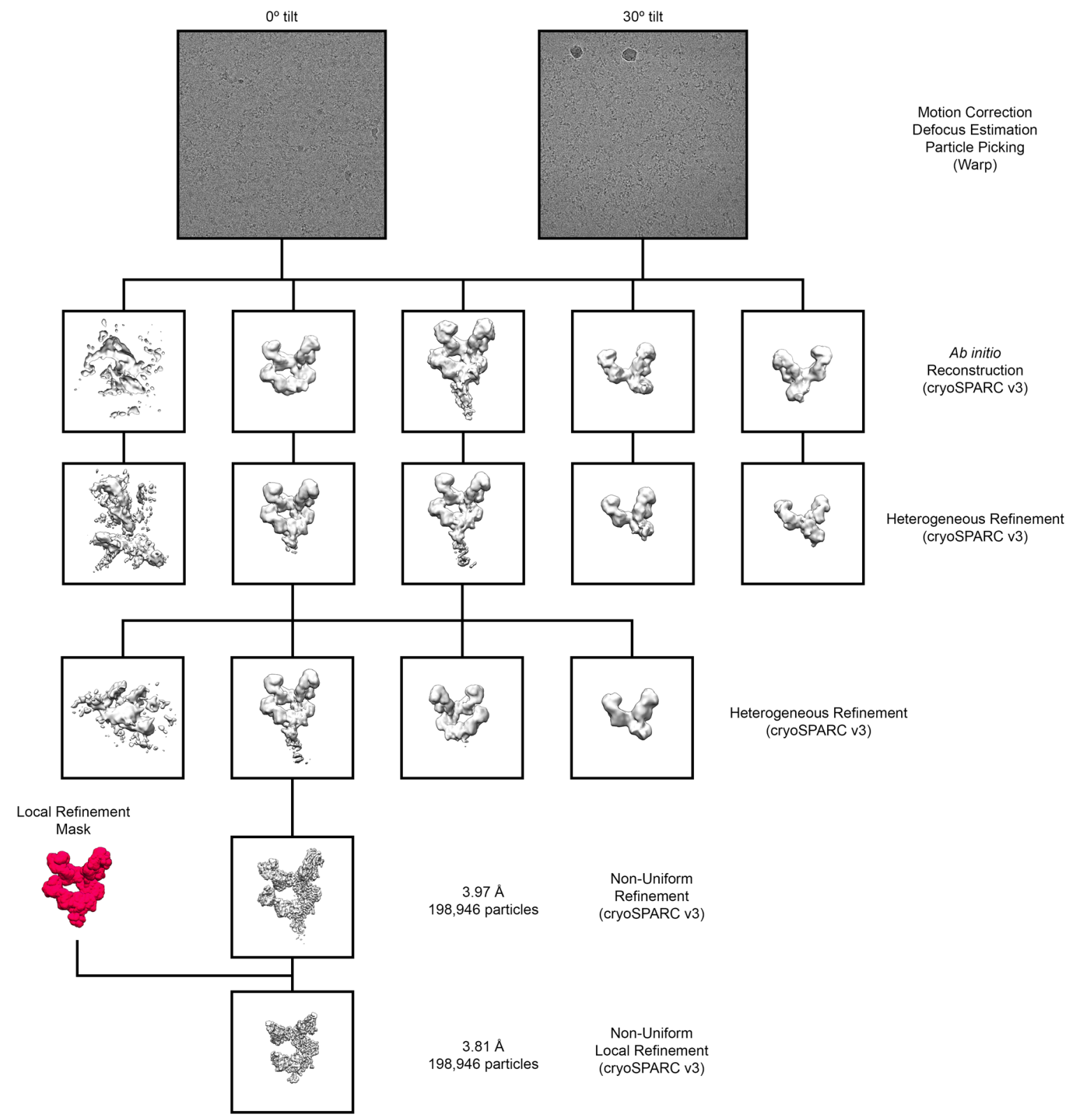

Supplementary Figure 6: Pentamer + 1-103 + 1-32 + 2-25 cryo-EM data processing workflow. 
bioRxiv preprint doi: https://doi.org/10.1101/2021.03.25.436804; this version posted March 25, 2021. The copyright holder for this preprint (which was not certified by peer review) is the author/funder, who has granted bioRxiv a license to display the preprint in perpetuity. It is made available under aCC-BY-NC-ND 4.0 International license.

Supplementary Figure 7

Motion Correction
Defocus Estimation

Defocus Estimation
Particle Picking

(Warp)
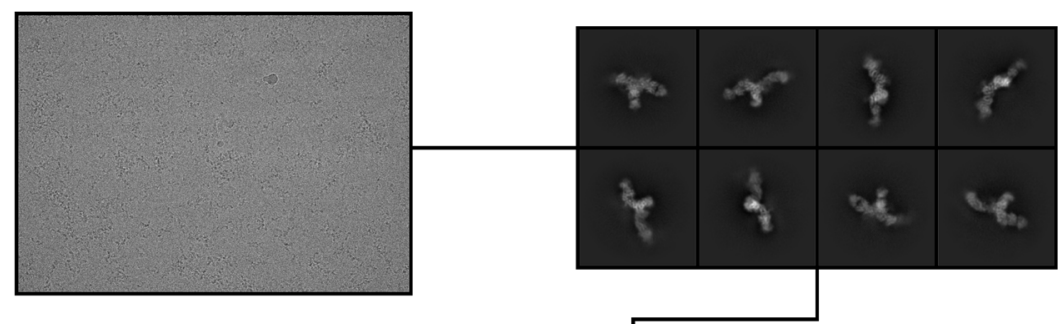

2D Classification

(cryoSPARC v2)

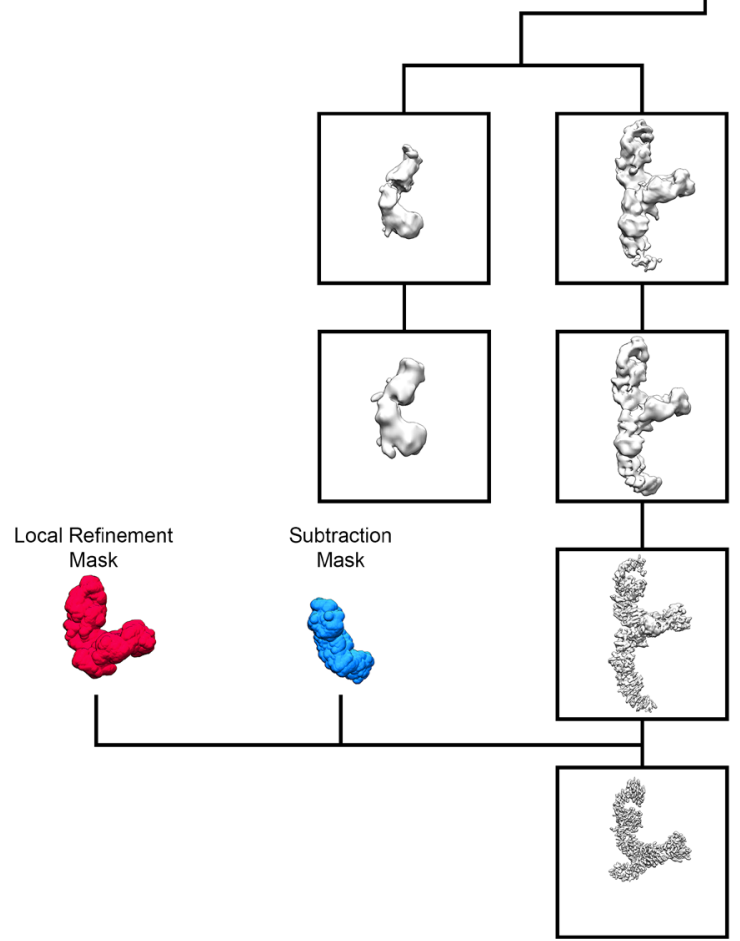

Ab initio

Reconstruction

(cryoSPARC v2)

Heterogeneous Refinement

(cryoSPARC v2)

$4.46 \AA$

249,802 particles

Non-Uniform

Refinement

(cryoSPARC v2)

$4.02 \AA$

249,802 particles

Non-Uniform Local Refinement (cryoSPARC v2)

Supplementary Figure 7: Pentamer $+2-18+8 \mathrm{I} 21$ cryo-EM data processing workflow. 
Supplementary Figure 8
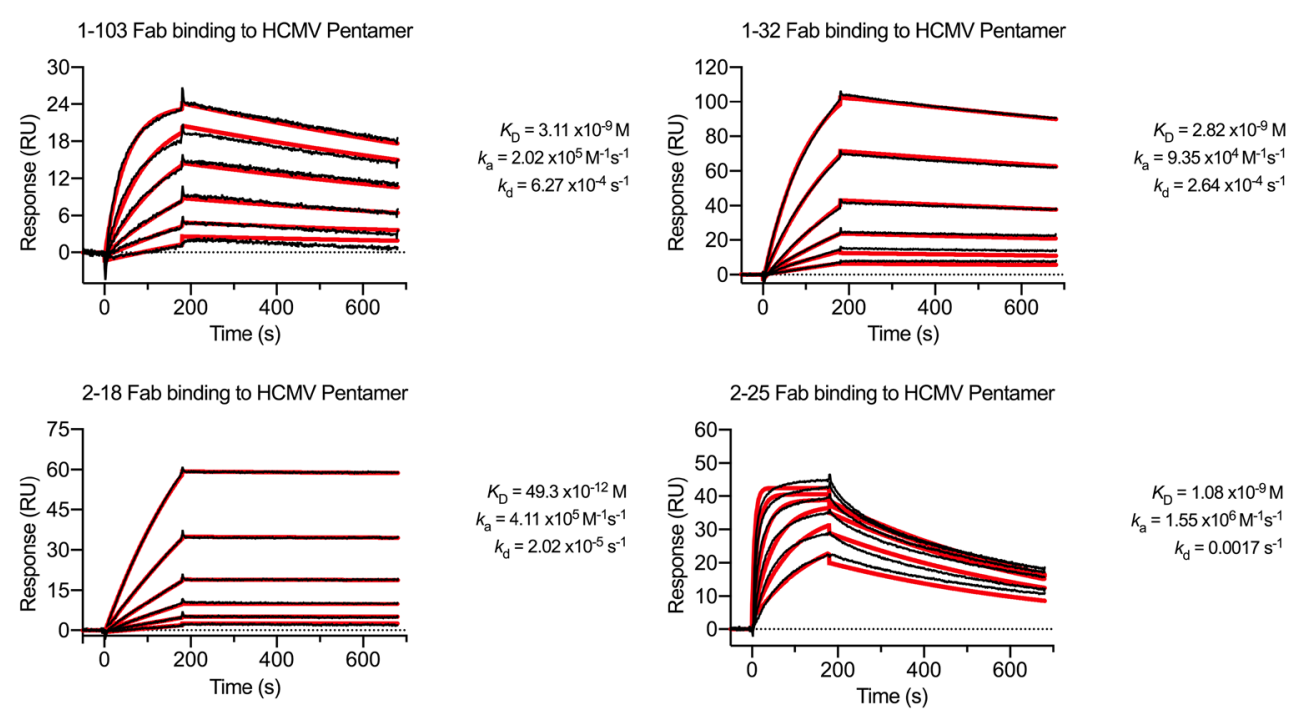

Supplementary Figure 8: Binding kinetics of Pentamer-directed antibodies. SPR sensorgrams showing binding of each of the four neutralizing Fabs are displayed, with data shown as black lines and the best fit of a 1:1 binding model shown as red lines. 
Supplementary Figure 9

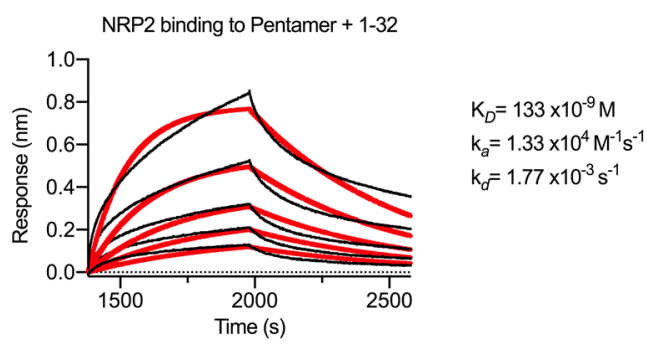

Supplementary Figure 9: NRP2 binding to Pentamer is partially disrupted by the presence of 132. Sensorgrams are shown for an experiment in which 1-32 IgG was immobilized to a BLI sensor, then used to capture Pentamer before being dipped into NRP2. Data for the association and dissociation of NRP2 are shown as black lines and the lines of best fit of a 1:1 binding model are shown as red lines. 
Supplementary Figure 10

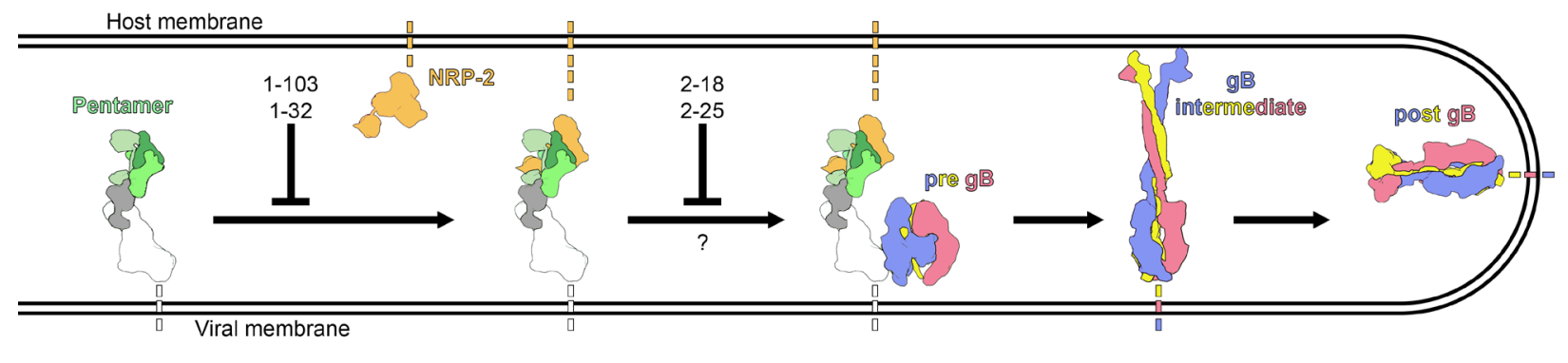

Supplementary Figure 10: Pentamer-directed mAbs neutralize via distinct mechanisms. A cartoon is shown depicting the infection of an endothelial or epithelial cell by HCMV. Pentamer is colored according to Fig. 1, NRP2 is colored orange and the three protomers of $\mathrm{gB}$ are colored red, blue and yellow. The stages of infection that mAbs 1-103, 1-32, 2-18 and 2-25 are predicted to disrupt are denoted by inhibition arrows. 


\section{Supplementary Table 1}

\begin{tabular}{|c|c|c|c|c|}
\hline & 1-103 Fab & 1-32 Fab & 2-18 Fab & 2-25 Fab \\
\hline \multicolumn{5}{|l|}{ Data collection } \\
\hline Facility & APS 19-ID & APS 19-ID & APS 19-ID & APS 19-ID \\
\hline Wavelength $(\AA)$ & 0.979 & 0.979 & 0.979 & 0.979 \\
\hline Space group & $P 2$ & $P 41212$ & $C 2$ & $P 43212$ \\
\hline \multicolumn{5}{|l|}{ Cell dimensions } \\
\hline a, b, c $(\AA)$ & $95.4,105.2,102.7$ & $65.7,65.7,191.9$ & $129.5,59.2,141.7$ & $180.9,180.9,138.9$ \\
\hline$\alpha, \beta, \gamma\left({ }^{\circ}\right)$ & $90.0,91.4,90.0$ & $90.0,90.0,90.0$ & $90.0,91.5,90.0$ & $90.0,90.0,90.0$ \\
\hline Resolution range $(\AA)$ & $52.62-1.90(1.93-1.90)$ & $54.20-2.10(2.16-2.10)$ & $65.02-2.80(2.95-2.80)$ & $69.89-2.51(2.57-2.51)$ \\
\hline$R_{\text {merge }}$ & $0.059(0.407)$ & $0.174(0.951)$ & $0.352(1.283)$ & $0.187(2.033)$ \\
\hline $\mathrm{CC} 1 / 2$ & $0.996(0.736)$ & $0.987(0.753)$ & $0.851(0.291)$ & $0.991(0.522)$ \\
\hline |/бl & $5.9(1.8)$ & $6.0(1.5)$ & $4.0(1.7)$ & $9.3(2.0)$ \\
\hline Completeness (\%) & $90.2(91.3)$ & $99.9(99.8)$ & $98.8(96.7)$ & $98.6(99.9)$ \\
\hline Redundancy & $2.0(2.0)$ & $7.2(6.6)$ & $4.1(3.8)$ & $6.8(7.1)$ \\
\hline \multicolumn{5}{|l|}{ Refinement } \\
\hline No. reflections & $143,434(14,383)$ & $25,398(2,476)$ & $26,469(2,446)$ & $77,206(7,680)$ \\
\hline $\mathrm{R}_{\text {work }} / \mathrm{R}_{\text {free }}(\%)$ & $19.1 / 21.7$ & $26.8 / 29.7$ & $22.1 / 25.5$ & $18.5 / 21.9$ \\
\hline \multicolumn{5}{|c|}{ No. non-hydrogen atoms } \\
\hline Protein & 12,781 & 3,226 & 6,726 & 9,633 \\
\hline Ligand/ion & 0 & 0 & 0 & 0 \\
\hline Water & 1,627 & 70 & 70 & 507 \\
\hline \multicolumn{5}{|l|}{ B-factors $\left(\AA^{2}\right)$} \\
\hline Protein & 28.7 & 62.9 & 27.0 & 39.9 \\
\hline Solvent & 38.8 & 49.7 & 27.1 & 42.9 \\
\hline \multicolumn{5}{|l|}{ R.m.s. deviations } \\
\hline Bond lengths $(\AA)$ & 0.007 & 0.013 & 0.011 & 0.011 \\
\hline Bond angles $\left(^{\circ}\right)$ & 1.33 & 1.41 & 1.46 & 1.23 \\
\hline \multicolumn{5}{|l|}{ Ramachandran (\%) } \\
\hline Favored & 98.4 & 95.7 & 97.3 & 98.1 \\
\hline Allowed & 1.6 & 4.3 & 2.7 & 1.9 \\
\hline Outliers & 0 & 0 & 0 & 0 \\
\hline PDB ID & 7LYV & $7 \mathrm{M} 1 \mathrm{C}$ & $7 \mathrm{KBA}$ & 7LYW \\
\hline
\end{tabular}

Supplementary Table 1: X-ray crystallographic data collection and refinement. 


\section{Supplementary Table 2}

Data collection and processing

Magnification (nominal)

Voltage $(\mathrm{kV})$

Electron exposure $\left(\mathrm{e}^{-} / \AA^{2}\right)$

Defocus range $(\mu \mathrm{m})$

Pixel size $(\AA)$

Symmetry imposed

Initial particles

Final particles

Map resolution $(\AA)$

Focused refinement resolution $(\AA)$

FSC threshold

\section{Refinement}

Initial model(s) used (PDB ID)

Model composition

Protein atoms

Protein B-factors (mean)

R.m.s. deviations

Bond lengths $(\AA)$

Bond angles $\left({ }^{\circ}\right)$

Validation

Molprobity score

Clashscore

Rotamer outliers (\%)

Ramachandran plot

Favored (\%)

Allowed (\%)

Outliers (\%)

EMRinger score

Data Availability

EMDB

PDB
Pentamer

+ NRP2

300

80

1.0-2.0

1.073

n/a (C1)

745,025

203,130

4.00

3.65

0.143

5V0B, 2QQK

6011

82.1

0.005

1.14

1.69

7.47

0.61

95.9

4.1

0.0

2.84

23629

$7 \mathrm{M} 22$
Pentamer

$+2-18+8121$

300
36
$1.0-3.0$
1.047

n/a (C1)

$1,489,510$

249,802

4.46

4.02

0.143

5VOB, 7KBA

6422

43.6

0.007

1.38

2.21

14.42

1.58

94.1

5.4

0.5

1.21

22788

7KBB
Pentamer

$+1-103+2-25+1-32$
300
36
1.1-2.4
1.075

$\mathrm{n} / \mathrm{a}(\mathrm{C} 1)$

834,092

198,946

3.97

3.81

0.143

5V0B, 7LYV, 7M1C, 7LYW

9163

83.1

0.006

1.38

1.84

8.91

0.89

94.7

5.3

0.0

1.80

23640

$7 \mathrm{M} 30$

Supplementary Table 2: Cryo-EM data collection and refinement. 